\title{
Long Noncoding RNA TUG1 Inhibits Tumor Progression through Regulating Siglec-15-Related Anti-Immune Activity in Hepatocellular Carcinoma
}

\author{
Yanyi Ren, ${ }^{1}$ Jiayi Lyu, ${ }^{2}$ Yaoguang Guo, ${ }^{3}$ Yuan Yao $\mathbb{D}^{4},{ }^{4}$ and Lin $\mathrm{Hu} \mathbb{D}^{5}$ \\ ${ }^{1}$ Department of Gastroenterology, Hospital of Chengdu University of Traditional Chinese Medicine, Chengdu, China \\ ${ }^{2}$ Department of Clinical Laboratory, Hospital of Chengdu University of Traditional Chinese Medicine, Chengdu, China \\ ${ }^{3}$ Department of Acupuncture and Moxibustion, Hospital of Chengdu University of Traditional Chinese Medicine, Chengdu, China \\ ${ }^{4}$ Department of Neurosurgery, Hospital of Chengdu University of Traditional Chinese Medicine, Chengdu, China \\ ${ }^{5}$ Department of Medical Imaging, Hospital of Chengdu University of Traditional Chinese Medicine, Chengdu, China
}

Correspondence should be addressed to Yuan Yao; yaoyuan5486@163.com and Lin Hu; candice_lin2021@163.com

Received 16 August 2021; Revised 11 December 2021; Accepted 13 December 2021; Published 21 February 2022

Academic Editor: Sainan Li

Copyright (C) 2022 Yanyi Ren et al. This is an open access article distributed under the Creative Commons Attribution License, which permits unrestricted use, distribution, and reproduction in any medium, provided the original work is properly cited.

Background. Hepatocellular carcinoma (HCC) is the second leading cause of cancer-related death, and its biology remains poorly understood, especially in regards to the immunosuppression induced by immune checkpoints, such as Siglec-15. Most cancer treatments composed of immune checkpoint inhibitors and oncogene-targeted drugs display a better therapeutic effect in the clinic, including tumor progression inhibition and immunosuppression breaks. However, two or more drugs will result in a greater possibility of adverse effects. Thus, a double-function target is necessary for developing antitumor drugs, such as RNAi therapy. Methods. The expression of TUG1, Siglec-15, and miRNAs was evaluated by qPCR, and protein expression was analyzed by western blotting. The immune responses were evaluated by a Jurkat-reporter gene assay, a $\mathrm{T}$ cell-induced cytotoxicity assay, and IFN- $\gamma /$ IL-2 release. The interactions among TUG1, Siglec-15, and miRNAs were verified by dualluciferase reporter, RNA immunoprecipitation, and RNA pull-down assays. CCK-8 and Transwell assays were used to determine tumor cell proliferation, migration, and invasion. Results. In HCC patients and cells, increased TUG1 levels were observed, positively regulating Siglec-15 expression. TUG1-induced Siglec-15 upregulation resulted in the suppression of the immune response of HCC cells. hsa-miR-582-5p directly targeted TUG1 and Siglec-15 mRNA, and ihsa-miR-582-5p knockout prevented the regulation of Siglec-15 induced by THU1. Changes in hsa-miR-582-5p expression negatively regulated Siglec-15 levels and immunosuppression but had no influence on TUG1 levels. siRNA knockdown of TUG1 effectively led to tumor progression inhibition and immune response improvement in HCC cells both in vitro and in vivo. Conclusion. TUG1 increases the Siglec-15 level in HCC cells as a sponge to hsa-miR-582-5p, resulting in enhanced immunosuppression. TUG1 knockdown induced by siRNA not only reduces immunosuppression but also suppresses tumor progression both in vitro and in vivo. These novel findings may provide a potential and appropriate target for RNAi therapy to develop drugs with dual antitumor activity.

\section{Introduction}

Hepatocellular carcinoma (HCC) is the second leading cause of cancer-related death and the sixth most prevalent cancer worldwide with increasing incidence in recent decades [1]. Although developments in surgical techniques have enhanced the prognosis of patients with HCC, the overall survival is still poor with a median survival of 9 months and a 3-year survival rate of $12.7 \%$ [2]. Thus, developing more reliable and novel strategies is required to improve treatment efficacy.

Recently, a new therapeutic strategy, RNA interference (RNAi), has received much attention. RNAi is a ubiquitous posttranscriptional gene silencing (PTGS) mechanism found in metazoan cells. mRNAs encoding proteins or other noncoding RNAs are inhibited by small duplex RNAs, such as 
short interfering RNAs (siRNAs) and microRNAs (miRNAs). Most RNAi drugs in preclinical or clinical trials use siRNA due to its high specificity compared to miRNAs [3]. Despite the challenge of RNAi delivery, liver-targeted RNAi delivery is relatively established using GalNAc-siRNA conjugates with several applications in clinical trials [4]. Thus, it is possible to treat HCC using the RNAi technology if there are appropriate target genes.

Recently, sialic acid-binding immunoglobulin-like lectin 15 (Siglec-15), one of the Siglec gene family members with a characteristic sialic acid-binding immunoglobulin-type lectin structure [5], has been demonstrated to be an immune checkpoint and called a next-generation immunooncology agent $[6,7]$. In contrast, the regulation of Siglec-15 in HCC is limited. Long noncoding RNAs (lncRNAs) are RNAs without protein-coding ability and are longer than nucleotides [8]. Taurine-upregulated gene 1 (TUG1) is a lncRNA that was first identified in taurine-treated mouse retinal cells [9]. Many reports have demonstrated that TUG1 knockdown inhibits cancer progression and suppresses tumor growth in many cancers, including HCC [10-13]. However, the regulatory role of TUG1 in immune checkpoints, such as PD-L1/PD1, CTAL-4, and Siglec-15, is still unknown, especially in HCC.

Here, we explored the function of TUG1 in HCC to regulate Siglec-15 as a sponge to target hsa-miR-582-5p, thereby downregulating Siglec-15 expression. In addition, we designed and screened siRNAs targeting the conserved region of TUG1 as RNAi drug candidates, which inhibited tumor growth and progression as well as improved $\mathrm{T}$ cellinduced antitumor immune activity both in vitro and in vivo. Thus, TUG1 may be an appropriate target for RNAi therapy in HCC.

\section{Methods and Materials}

2.1. Bioinformatics Strategy for Identification of IncRNAs. Briefly, we first identified the lncRNAs with abnormal expression in HCC according to the Lnc2Cancer database (http://biobigdata.hrbmu.edu.cn/lnc2cancer/) and found 513 lncRNAs, including 340 upregulated lncRNAs. We then analyzed the correlation between Siglec-15 and the 340 lncRNAs using the StarBase database (http://starbase.sysu .edu.cn/starbase2/index.php). Only 45 lncRNAs showed correlation, and 25 of these showed a positive correlation with Siglec-15. The reason for focusing on $\operatorname{lncRNAs}$ upregulated in HCC and positively correlated with Siglec-15 was to develop siRNA drugs inhibiting Siglec-15 through downregulating lncRNAs, which may have other synergistic effects. We also used TCGA to confirm the remaining 25 lncRNAs, excluding 8 lncRNAs. Thus, we hypothesized that these remaining lncRNAs play a significant role in HCC progression and development, and we also hypothesized that Siglec-15 regulation influences the survival of HCC patients.

2.2. Cell Culture. The LO-2 human normal hepatic cell line and the QGY-7701 and HUH7 HCC cell lines were obtained from ATCC. The HepG2, Bel-7404, SK-Hep-1, and Hep3B HCC cell lines were obtained from the National Collection of Authenticated Cell Cultures (NCAC) in China. Primary human hepatocytes ( $\mathrm{HH}$, mixed donor) were obtained from Lonza (HUCS10P). Cell lines were cultured with Eagle's minimum essential medium (EMEM, Gibco) supplemented with $10 \%$ fetal bovine serum (FBS, Gibco). HH cells were cultured in $\mathrm{HCM}^{\mathrm{TM}}$ Hepatocyte Culture Medium BulletKit ${ }^{\mathrm{TM}}$ (CC-3198, Lonza). All cells were incubated at $37^{\circ} \mathrm{C}$ and $5 \%$ $\mathrm{CO}_{2}$. hsa-miR-582-5p knockout (miRKO) cell lines were generated by GenePharma with the CRISPR/Cas9 system.

2.3. Transfection and Transduction. The siRNAs, miRNAs, miRNA inhibitors and negative controls were synthesized by Sangon Biotech (China). Plasmids for transient overexpression of TUG1 in the pCDA3.1 backbone and lentiviruses containing TUG1-IRES-EGFP, TUG1, and sh-TUG1 were generated by GenePharma. The integration titer of lentivirus was $1 \times 10^{8} \mathrm{TU} / \mathrm{ml}$ (quantified by GenePharma). Transfection was performed when cells reached $50-60 \%$ confluence using Lipofectamine 3000 reagents (Thermo Fisher Scientific) according to the manufacturer's instructions. Lentiviral transduction was performed when cells reached 70-80\% confluence with a MOI of 20. Twenty-four hours after transduction, the medium was removed and replaced with fresh medium containing $2 \mu \mathrm{g} / \mathrm{ml}$ puromycin (Sigma), and cells were cultured for two weeks. Stable cell lines were maintained by culturing with medium containing $2 \mu \mathrm{g} / \mathrm{ml}$ puromycin.

\subsection{Quantitative Polymerase Chain Reaction (qPCR).} miRNA was extracted by TRIzol reagent (Sigma), and reverse transcription was performed by an One-Step miRNA cDNA Synthesis Kit (Takara). mRNA was also obtained using TRIzol reagent and transcribed by a PrimeScript RT reagent kit (Takara). mRNA expression was measured by the TB Green ${ }^{\circledR}$ Fast qPCR Mix (Takara). The measurement of miRNAs was performed using stem-loop qRT-PCR (TaqMan method) (Applied Biosystems). The primers and probes for miRNA qPCR were obtained from TaqMan ${ }^{\circledR}$ MicroRNA Assays (Applied Biosystems). The following qPCR primers were used: Siglec-15-F, $5^{\prime}$-TTCTCCCGACA GGCTCATTT-3'; Siglec-15-R, 5'-TGTGCACCTCTGTG TTGAGC-3'; TUG1-F, $5^{\prime}$-ACGACTGAGCAAGCACTAC C-3 ${ }^{\prime}$; TUG1-R, $5^{\prime}$-CTCAGCAATCAGGAGGCACA-3'; GAPDH-F, AGGTCGGAGTCAACGGATTT; and GAPDH-R, TGGAATTTGCCATGGGTGGA.

2.5. Western Blot Analysis. Cells were lysed with RIPA buffer (Sangon), and proteins were quantified using a BSA Kit (Beyotime, Shanghai, China). Tumor proteins were extracted by a One-Step Animal Tissue Active Protein Extraction kit (Sangon). Protein $(20 \mu \mathrm{g})$ was loaded and separated by $12 \%$ sodium dodecyl sulfate-polyacrylamide gel electrophoresis (SDS-PAGE) (Fdbio Science, Hangzhou, China) at $80 \mathrm{~V}$ for $30 \mathrm{~min}$ and then $120 \mathrm{~V}$ for $60 \mathrm{~min}$ followed by transfer to polyvinylidene fluoride (PVDF) membranes (Millipore) at $300 \mathrm{~mA}$ for $90 \mathrm{~min}$. After blocking with $5 \%$ fat-free milk for $1 \mathrm{~h}$, the membranes were incubated with primary antibodies overnight at $4^{\circ} \mathrm{C}$. The following primary antibodies were used: anti-Siglec-15 (1:1000, Santa 
Cruz), anti-GAPDH (1:5000, Sino Biological), anti-AGO2 $(1: 20$, Abcam), anti-C-caspase3 $(1: 1000$, Abcam), anticaspase3 (1:1000, Abcam), and anti-Bcl-2 (1:1000, Abcam). After incubation with HRP-conjugated antibodies $(1: 5000$, Sino Biological) for $2 \mathrm{~h}$ at room temperature, an ECL kit (Fdbio Science) was used for visualization of protein bands.

2.6. Jurkat-Based Reporter Gene Assay (RGA). The luc2P/ NFAT-RE/Hygro sequence was obtained from the pGL4.30-luc2P/NFAT-RE/Hygro vector (Promega). Lentivirus containing this sequence was generated by GenePharma with an integration titer of $1 \times 10^{8} \mathrm{TU} / \mathrm{ml}$. Jurkat cells $($ ATCC) were transduced with this lentivirus $(\mathrm{MOI}=20)$ at $3 \times 10^{5}$ cells $/ \mathrm{ml}$ with RPMI 1640 (Gibco) containing $10 \%$ FBS and $8 \mu \mathrm{g} / \mathrm{ml}$ polybrene. Twenty-four hours after transduction, the medium was removed and replaced with fresh medium containing $100 \mu \mathrm{g} / \mathrm{ml}$ hygromycin, and cells were cultured for two weeks to generate stable cells. The cell line was maintained in medium containing $100 \mu \mathrm{g} / \mathrm{ml}$ hygromycin.

For the RGA assay, $2.5 \times 10^{4}$ cells were plated into 96well plates $(100 \mu \mathrm{l} /$ well $)$ in medium containing other reagents. After $24 \mathrm{~h}$, the medium was removed, and $1.5 \times$ $10^{5}$ engineered Jurkat cells were added to wells with $100 \mu \mathrm{l}$ of medium containing the OKT3 CD3 agonist antibody (PeproTech). Cells were incubated for $8 \mathrm{~h}$ at $37^{\circ} \mathrm{C}$ and $5 \%$ $\mathrm{CO}_{2}$. Subsequently, $100 \mu \mathrm{l}$ of Promega Bio-GloTM Luciferase Assay was added to each well. After shaking the plate, a plate reader was used to measure the relative luciferase units (RLUs).

2.7. CD3+ T Cell-Induced Cytotoxicity Assay. Fresh human peripheral blood mononuclear cells (PBMCs) were purchased from Sailybio (Shanghai, China), and CD3+ T cells were separated by CD3 microbeads (Miltenyi Biotec). Tumor cells $\left(2 \times 10^{4}\right.$; target $)$ were plated in triplicate into 96 -well plates and cocultured with $\mathrm{CD} 3+\mathrm{T}$ cells (Effector) at a ratio of $2: 1$ (effector:target (E:T)) in 1640/RPMI medium containing $10 \%$ FBS. After incubation for $8 \mathrm{~h}$, lactate dehydrogenase (LDH) from damaged cells was measured by a LDH Cytotoxicity Assay Kit (Beyotime). The absorbance was read with a microplate reader (Biotech Instruments Inc. USA) at $490 \mathrm{~nm}$. The percent of cytotoxicity was calculated according the following formula: cytotoxicity $\%=($ samples $-\mathrm{E}$ only blank $) /($ T only blank $) \times 100$.

2.8. IFN- $\gamma$ and IL-2 Analysis. Supernatants were collected from the cytotoxicity assay, and the concentrations of interleukin-2 (IL-2) and interferon- $\gamma$ (IFN- $\gamma$ ) were measured using ELISA kits (Abcam).

2.9. Dual-Luciferase Reporter Assay. Luciferase activity assays were performed as previously described [14]. Briefly, HEK-293T cells were plated into a 96-well plate at $1 \times 10^{5}$ cells/well and incubated for $24 \mathrm{~h}$ at $37^{\circ} \mathrm{C}$ and $5 \% \mathrm{CO}_{2}$. Cells were then cotransfected with $200 \mathrm{ng}$ of luciferase reporter vectors (Promega) containing target sequences and miRNAs. After $48 \mathrm{~h}$, cells were lysed, and the luciferase activity was measured with the Dual-Luciferase Reporter Assay System (Promega).

2.10. RNA Immunoprecipitation (RIP). The RIP assay was performed using the Magna RIP RNA-Binding Protein Immunoprecipitation Kit (Millipore). The lysate from $3 \times 10^{7}$ cells was prepared with $100 \mu \mathrm{l}$ of RIP lysis buffer. After centrifugation, the supernatant was collected and incubated with RIP wash buffer, and the subsequent procedures were performed according to the manufacturer's instructions. The following antibodies were used for the RIP assay: anti-AGO2 (1:20) and control IgG $(1: 20)$ (Abcam). qPCR was used to detect enriched RNA.

2.11. RNA Pull-Down. Briefly, $50 \mathrm{nM}$ biotinylated hsa-miR582-5p (GenePharma) was transfected into Hep3B cells for $24 \mathrm{~h}$ by Lipofectamine 3000 reagents (Thermo Fisher Scientific). Then $1 \times 10^{7}$ cells were harvested and lysed in lysis buffer, and the cell lysates were incubated with washed streptavidin magnetic beads (Life Technologies) at room temperature for $2 \mathrm{~h}$. Beads were washed briefly three times, and TRIzol reagent (Invitrogen) was used to extract RNA. The coprecipitated RNA was analyzed by qPCR.

2.12. Flow Cytometry. To detect the GFP signal, cells were analyzed using a FACSCano II instrument (BD Biosciences) after washing once with PBS. Apoptosis assays were performed using an Annexin V-FITC/PI apoptosis detection kit (Beyotime). Briefly, $1 \times 10^{5}$ cells were incubated with $200 \mu \mathrm{l}$ of buffer containing $5 \mu \mathrm{l}$ of Annexin V-FITC and $10 \mu \mathrm{l}$ of PI for $20 \mathrm{~min}$ at RT. Cells were then immediately placed on ice. Cells were washed once with PBS and analyzed on a FACSCano II instrument (BD Biosciences). The data were analyzed by FlowJo.

2.13. Cell Proliferation Assay. Cell proliferation was determined using a Cell Counting Kit-8 (CCK-8, Beyotime) assay according to the manufacturer's instructions. Briefly, cells were plated into 96 -well plates at $5 \times 10^{3}$ cells per well and cultured for $24 \mathrm{~h}$. siRNAs or miRNAs were then transiently transfected into cells and incubated for 24, 48, 72, 96, and $120 \mathrm{~h}$. At each time point, $10 \mu \mathrm{l}$ of CCK8 solution was added to each well and incubated for $2 \mathrm{~h}$ at $37^{\circ} \mathrm{C}$. The absorbance was measured at $450 \mathrm{~nm}$ by a microplate reader. All experiments were repeated at least three times.

2.14. 5-Ethynyl-2-Deoxyuridine (EdU) Assay. Cells were labeled with $100 \mu \mathrm{M}$ EdU-labeling medium (RiboBio, China) at $1 \times 10^{4}$ cells/well in 96 -well plates. After $12 \mathrm{~h}$ or $24 \mathrm{~h}$, cells were then treated with $4 \%$ paraformaldehyde and $0.5 \%$ Triton X-100 for $30 \mathrm{~min}$ followed by incubation with an anti-EdU working solution for $1 \mathrm{~h}$. Nuclei were stained with DAPI. Images were acquired using a fluorescence microscope.

2.15. Migration and Invasion Assay. For the migration assay, $2 \times 10^{5}$ cells in $300 \mu \mathrm{l}$ of serum-free medium were plated into the upper Transwell chamber, and $0.5 \mathrm{ml}$ of medium containing 30\% FBS was added to the lower chamber. After $48 \mathrm{~h}$, cells on the underside of the membrane were fixed 
and stained with crystal violet solution (Beyotime) for $2 \mathrm{~h}$ at $37^{\circ} \mathrm{C}$. Images were acquired using a microscope.

For the invasion assay, the steps were similar to those of the migration assay, except for one step. Before cell seeding, Matrigel (Corning) was coated onto the membrane on the upper Transwell chamber for $1 \mathrm{~h}$ at $37^{\circ} \mathrm{C}$.

2.16. Xenograft Model. Six-week-old NCG mice were obtained from GemPharmatech (Jiangsu, China). Mice were randomly divided into 4 groups $(n=6)$ after adopting the new environment for 2 weeks. Cells $\left(1 \times 10^{7}\right.$ cells/mouse) were subcutaneously injected into the back region of the mouse. At the same time, $100 \mu \mathrm{l}$ of PBS with or without PBMCs $\left(1 \times 10^{7}\right)$ was injected into the mouse via the tail vein. Two weeks after xenografting, in which the tumor volume reached $100-200 \mathrm{~mm}^{3}$, cholesterol-coupled siRNAs (GenePharma) were intratumorally injected into the tumor every 4 days for 20 days. The tumor volume was measured every 4 days after the first siRNA injection using a Vernier caliper, and the tumor volume was calculated with the following formula: volume $=$ length $\times$ width $^{2} / 2$. Tumor weight was determined at 30 days after the first siRNA injection.

All methods were performed following the guidelines and regulations of the Hospital of Chengdu University of Traditional Chinese Medicine Animal Care and Use Committee and the National Institutes of Health.

2.17. Statistical Analysis. Data were analyzed with the Student's $t$ test using Prism (version 5; GraphPad Software). Comparisons of multiple groups were performed using one-way ANOVA followed by Tukey's multiple comparisons test. All data were assessed for normality of distribution using the Shapiro-Wilk test. The results are expressed as the mean \pm SD (ns: $P>0.05 ;^{*}: P<0.05 ;^{* *}: P<0.01$; and ${ }^{* * *}$ : $P<0.005$. Values were considered statistically significant if $P<0.05)$.

\section{Results}

3.1. TUG1 Functions as a Mediator by Regulating Siglec-15 in HCC Cells. Siglec-15 has been identified as a new immune checkpoint and is considered a next-generation immunooncology agent $[6,7]$. Considering the importance of Siglec-15 in cancer, we explored its regulatory role in HCC, especially in lncRNA-controlled pathways. Therefore, we screened potential lncRNAs through bioinformatics methods following the workflow shown in Figure 1(a). From TCGA database, we found 2 lncRNAs, TUG1 and MCM3AP-AS1, that affected the survival curve in HCC (TUG1, $P=0.024$; and MCM3AP-AS1, $P=0.0098)$. Compared to the 32 search results of MCM3AP-AS1 plus Cancer in PubMed, 262 search results of TUG1 plus Cancer showed a clearer regulatory mechanism in tumor development. Thus, we focused on TUG1 as our target. The clinical expression and correlation with Siglec-15 of TUG1 are shown in Figures 1(b) and 1(c) (data from TCGA and analyzed by Pan-Cancer program in the Starbase web site).

We next analyzed the TUG1 expression in six HCC cell lines (HepG2, QGY-7701, Bel-7407, SK-Hep-1, Hep3B, and
HUH7), the LO-2 human normal hepatic cell line, and the $\mathrm{HH}$ primary human hepatocyte line. A significantly higher level of TUG1 was present in the HCC cell lines compared to the $\mathrm{HH}$ and LO-2 cells with the highest expression in Hep3B cells (Figure 1(d)). Consistently, a similar tendency was found for Siglec-15 expression in these cells at both the mRNA and protein levels (Figures $1(\mathrm{e})-1(\mathrm{~g})$ ). A positive correlation between TUG1 and Siglec-15 expression was identified as shown in Figures 1(d) and 1(e). We constructed stable cell lines with TUG1 overexpression or TUG1 knockdown using HUH7 (low endogenous TUG1 expression) and Hep3B (high endogenous TUG1 expression). In both Hep3B and HUH7 cells, high TUG1 expression resulted in the upregulation of Siglec-15 expression, and shRNA-induced TUG1 knockdown led to Siglec-15 downregulation, including both the mRNA and protein levels (Figures $1(\mathrm{~h})-1(\mathrm{k})$ ).

Moreover, we performed a series of assays to evaluate the anti-immune response in engineered Hep3B and HUH7 cells. The Jurkat-based RGA assay is a method to estimate the signal strength of $\mathrm{T}$ cell activity. Jurkat is a $\mathrm{T}$ cell line that can be activated by tumor cells, but it lacks a cytotoxic effect. Thus, the NF- $\kappa \mathrm{B}$-controlled luciferase expression in Jurkat cells reflects the signal strength excited by tumor cells, stimulating the $\mathrm{T}$ cell response. The RGA results demonstrated higher luciferase activity in cells overexpressing TUG1 but lower luciferase activity in TUG1-deficient cells, indicating that TUG1-induced Siglec-15 regulation affects the signal strength in the $\mathrm{T}$ cell response (Figures $1(\mathrm{l})$ and $1(\mathrm{~m})$ ). We also performed $\mathrm{T}$ cell-induced cytotoxicity in engineered Hep3B and HUH7 cells to further explore the role of TUG1 in the immunoreaction. We found elevated cytotoxicity in TUG1 knockdown cells and an adverse effect in TUG1-overexpressing cells (Figures $1(n)$ and 1(o)). Similar results were observed for the levels of IFN- $\gamma$ and IL-2, cytokines secreted by $\mathrm{T}$ cells to execute cytotoxicity (Figures 1(p)-1(s)).

These results illustrated that TUG1 functions as a negative regulator in the $\mathrm{T}$ cell-induced immune response by regulating Silgec-15 expression.

3.2. hsa-miR-582-5p Is Responsible for the Siglec-15 Regulation Induced by TUG1. One of the common mechanisms by which lncRNAs regulate downstream genes is through a lncRNA-miRNA-mRNA ceRNA network. Thus, we hypothesized that there was a miRNA between TUG1 and Siglec-15. After intersecting the predicted miRNAs targeting TUG1 and Siglec-15 by the TargetScan and starBase databases, we found that a total of 8 miRNAs had the potential to target both of them simultaneously (Figure 2(a)).

We subsequently screened those miRNAs using a luciferase reporter assay. The results showed that hsa-miR-224$3 \mathrm{p}$ and hsa-miR-582-5p could target the $3^{\prime}$-URT of Siglec15, while hsa-miR-512-3p, hsa-miR-4761-5p, hsa-miR-522$3 \mathrm{p}$, and hsa-miR-582-5p could target TUG1 (Figures 2(b) and $2(\mathrm{c})$ ). hsa-miR-582-5p was the only miRNA capable of targeting TUG1 and Siglec-15 simultaneously. We also found a negative correlation between hsa-miR-582-5p and Siglec-15 expression in TCGA database, but hsa-miR-582$5 \mathrm{p}$ showed no correlation with TUG1, indicating sponge 
LncRNA

Purpose

Database

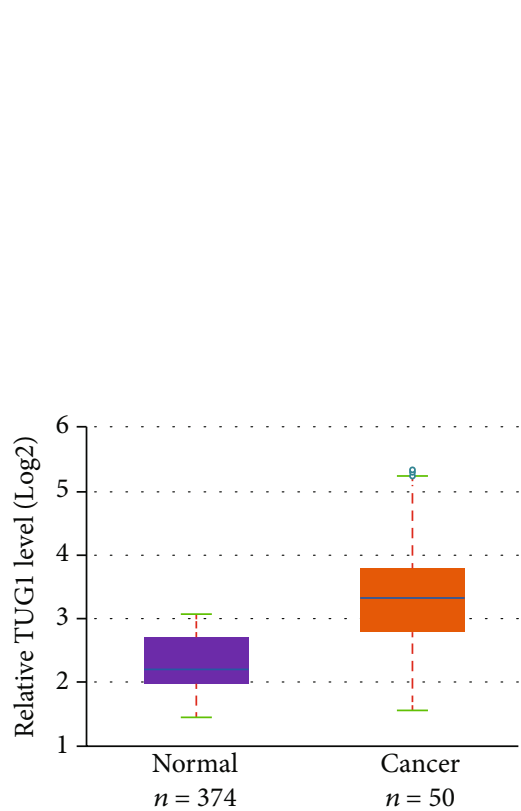

(b)

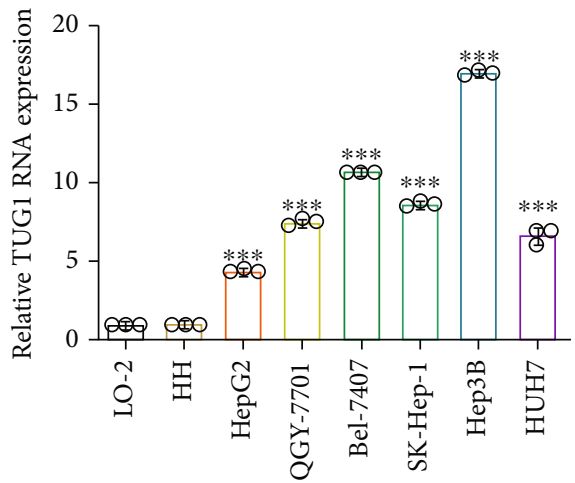

(d)

45
25

17

2

\begin{abstract}
Abnormal expression Up-regulation Correction with S-15 Positive-correction
\end{abstract}
Confirm of expression in TCGA

Comfirm of survive curve in TCGA

(a)

Satrbase

Satrbase

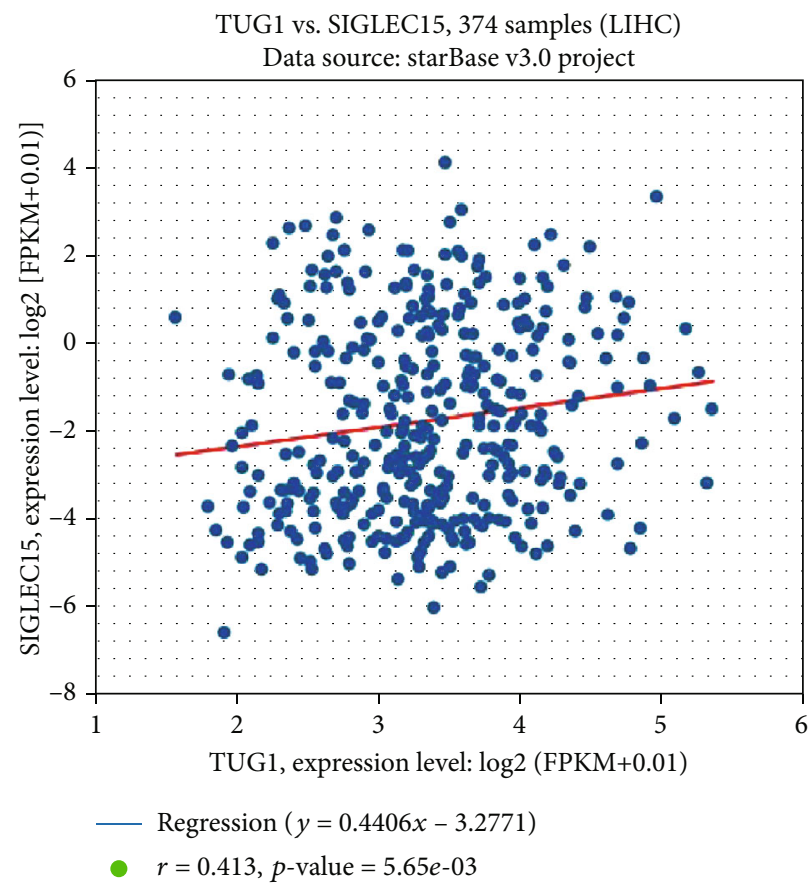

(c)

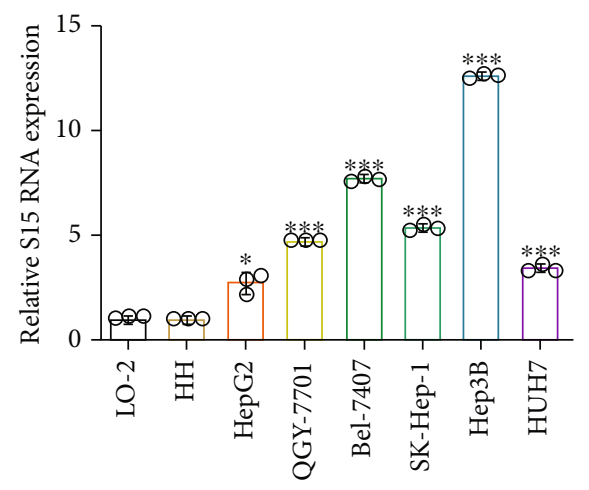

(e)

Figure 1: Continued. 


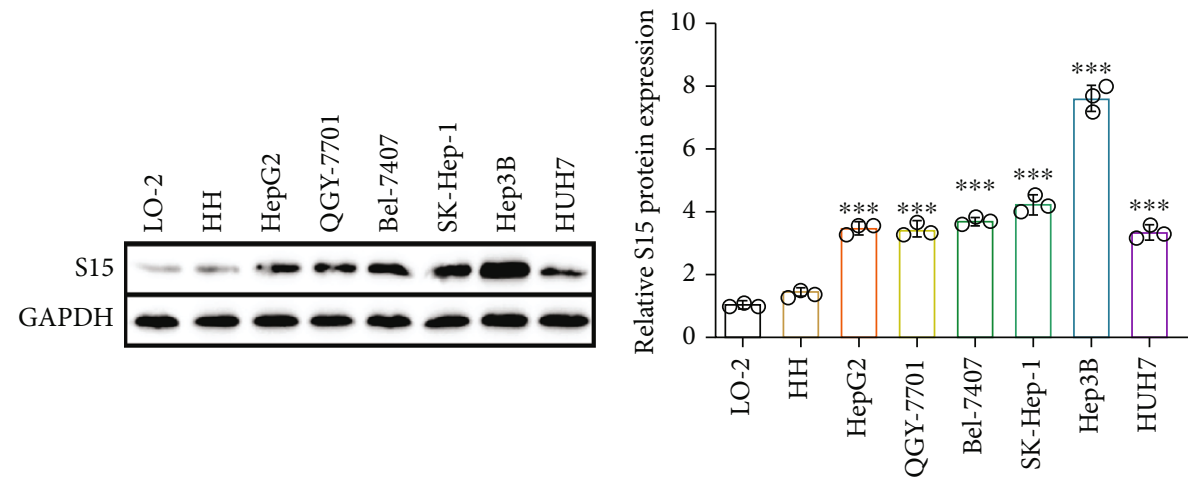

(f)
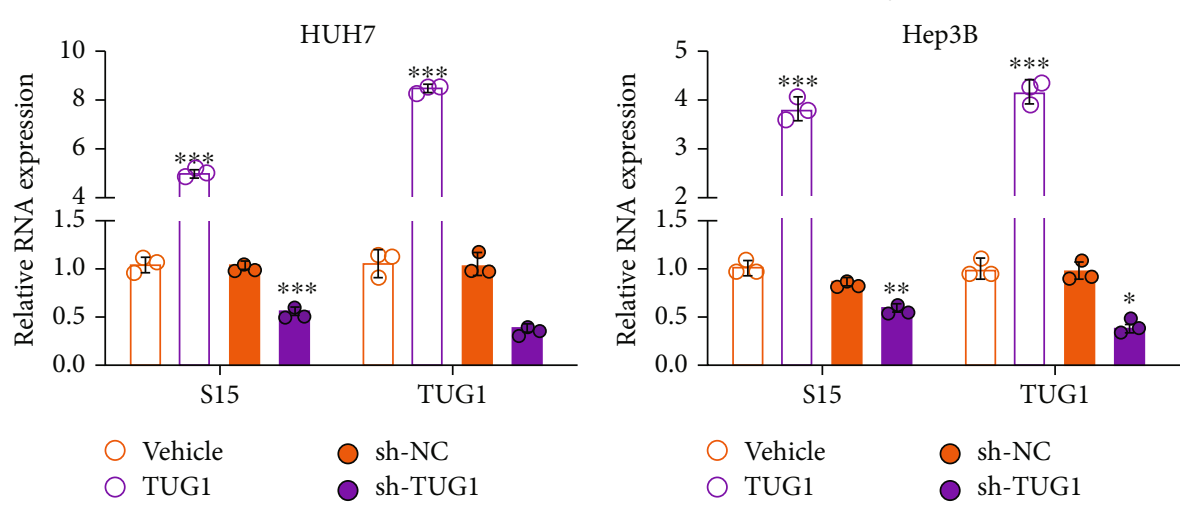

(h)

(i)
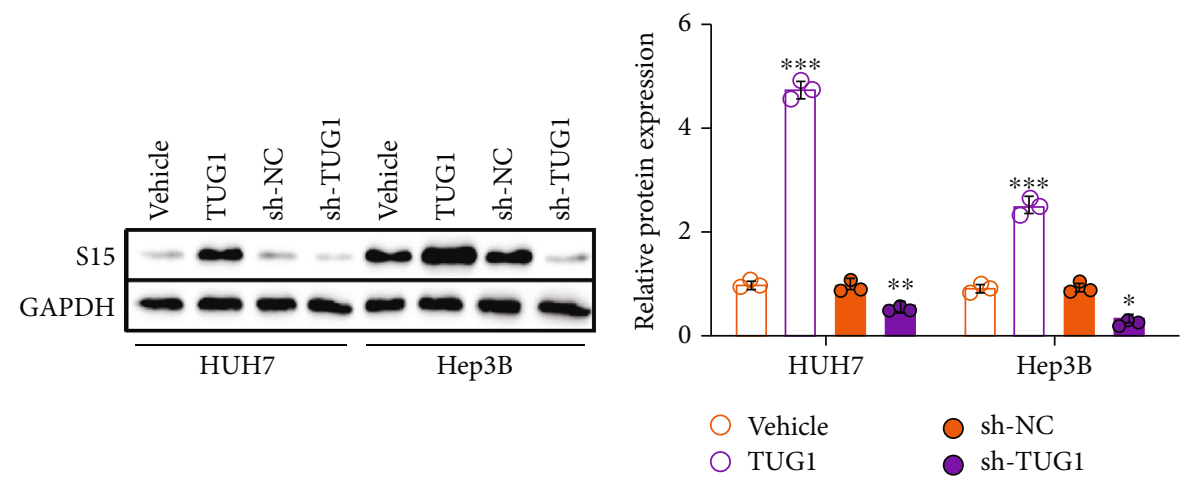

(j)

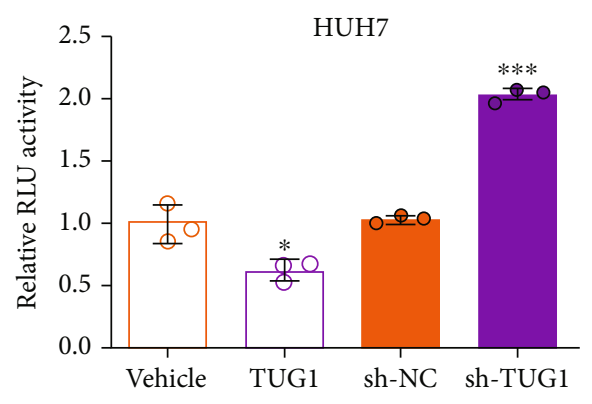

(l)

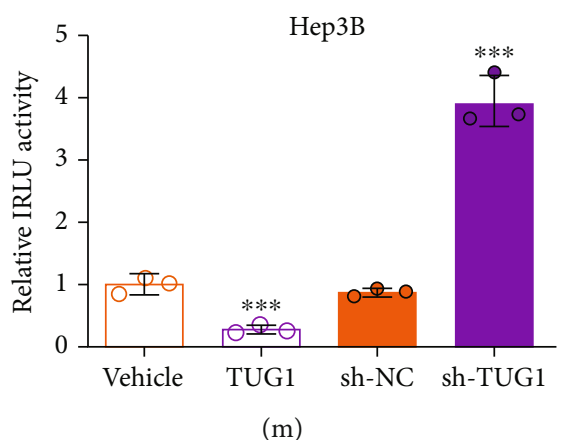

Figure 1: Continued. 


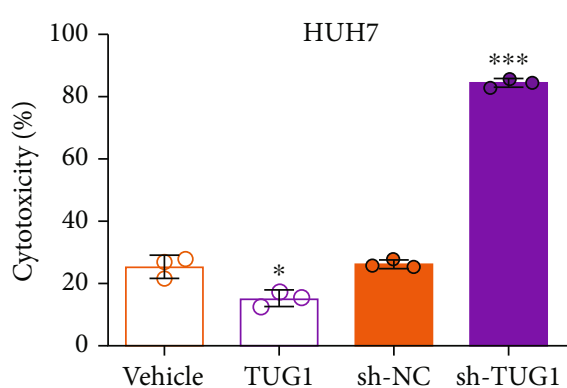

(n)

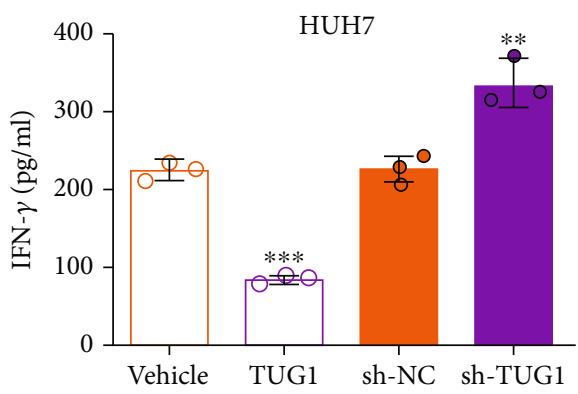

(p)

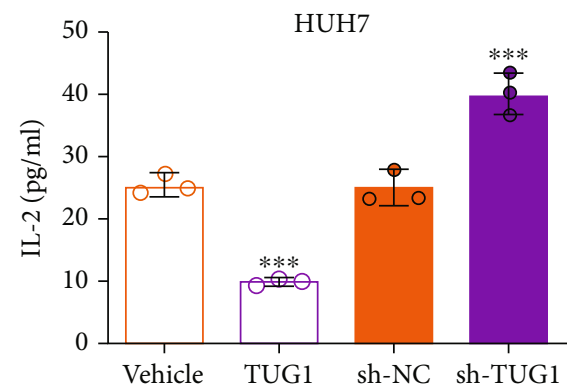

(r)

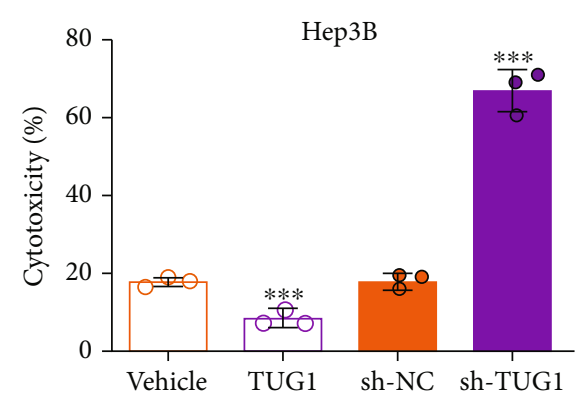

(o)

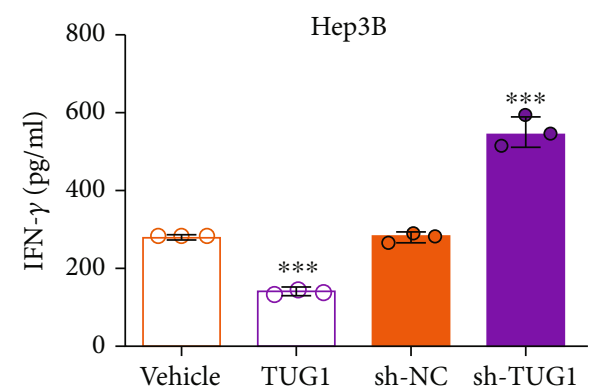

(q)

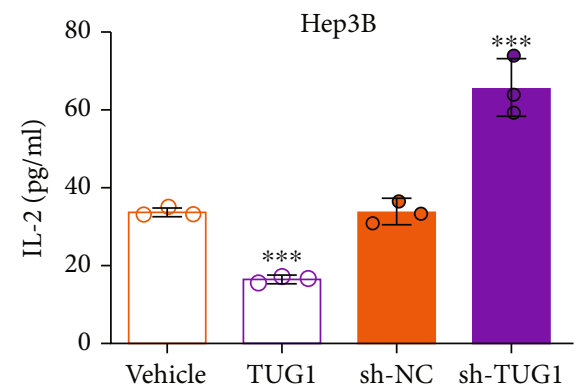

(s)

FIgURe 1: TUG1 is elevated in HCC cells and controls the Siglec-15 expression. (a) Schematic diagram of lncRNAs screen by bioinformation. (b) TUG1 expression in HCC patients from TCGA database. (c) Coexpression relationship between TUG1 and Siglec-15 in clinical data from TCGA database. (d) TUG1 expression, (e) Siglec-15 mRNA expression, and (f, g) protein expression in different HCC cell lines (HepG2, QGY-7701, Bel-7407, SK-Hep-1, Hep3B, and HUH7), human normal hepatic cell line LO-2, and primary human hepatocytes HH. (h, i) Siglec-15 mRNA expression and (j, k) protein expression in HUH7 and Hep3B cells treated with TUG1 overexpression or knockdown. (l, m) Relative luciferase activity of Jurkat-RGA in HUH7 and Hep3B cells treated with TUG1 overexpression or knockdown. ( $\mathrm{n}, \mathrm{o}) \mathrm{T}$ cell-induced cytotoxicity cocultured with HUH7 or Hep3B cells treated with TUG1 overexpression or knockdown. (p, q) IFN- $\gamma$ and $(\mathrm{r}, \mathrm{s})$ IL-2 secreted by T cells from $(\mathrm{n}, \mathrm{o})$. The data are presented as the means \pm SD, $n$ $=3$ experiments in $(\mathrm{d}-\mathrm{s}) .{ }^{*} P<0.05,{ }^{* *} P<0.01,{ }^{* * *} P<0.005$. S15: Siglec-15.

activity between TUG1 and hsa-miR-582-5p (Figures $2(\mathrm{~d})$ and 2(e)). The predicted binding format is shown in Figure 2(f). We also performed a luciferase assay using mutant groups (Mut) to confirm the interaction. The luciferase activity of TUG1-WT or Siglec-15-WT was inhibited in the presence of hsa-miR-582-5p with no difference in the Mut groups, suggesting that hsa-miR-582-5p targets TUG1 and the $3^{\prime}$-UTR of Siglec-15 simultaneously (Figures $2(\mathrm{~g})$ and $2(\mathrm{~h})$ ).

Furthermore, the RIP assay showed that Siglec-15 mRNA, TUG1, and hsa-miR-582-5p were enriched and coprecipitated with AGO2 protein (Figure 2(i)), indicating that they are involved in the AGO2 complex. We then used an RNA pulldown assay to investigate the interaction among Siglec-15 mRNA, TUG1, and hsa-miR-582-5p. In Hep3B cells, a biotin-labeled hsa-miR-582-5p pulled down TUG1 and also pulled down Siglec-15 mRNA (Figure 2(j)). These findings demonstrated that hsa-miR-582-5p directly and simultaneously binds to TUG1 and the $3^{\prime}$-UTR of Siglec-15. Moreover, we also found that the hsa-miR-582-5p-induced reduction of luciferase activity of the wild-type $3^{\prime}$-UTR in the Siglec-15 groups was transiently reversed by TUG1 cotransfection (Figure $2(\mathrm{k})$ ) without any changes in the mutant groups. Together, these results indicated that TUG1 may sponge hsa-miR-582-5p to regulate Siglec- 15 expression.

\section{3. hsa-miR-582-5p Regulates the T Cell-Induced Immune} Response of HCC Cells. Interestingly, a GEO analysis (GPL8179) has shown that hsa-miR-582-5p has a 0.53 -fold 

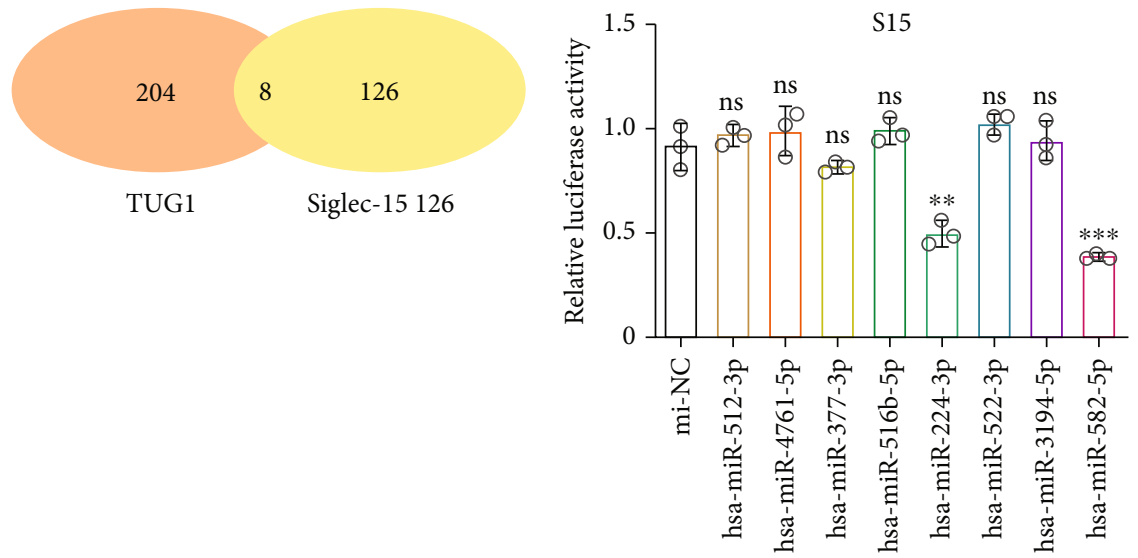

(a)

(b)

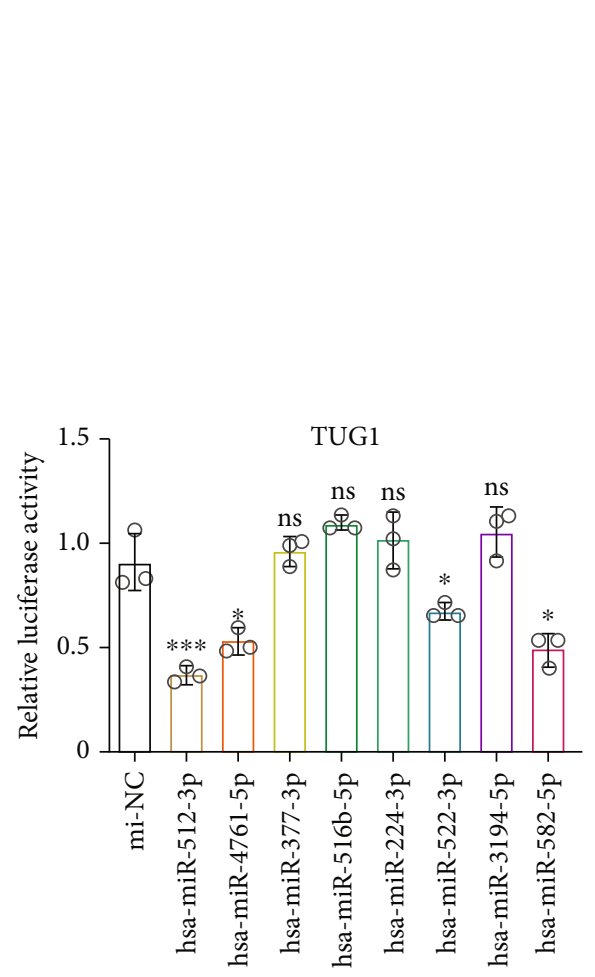

(c)

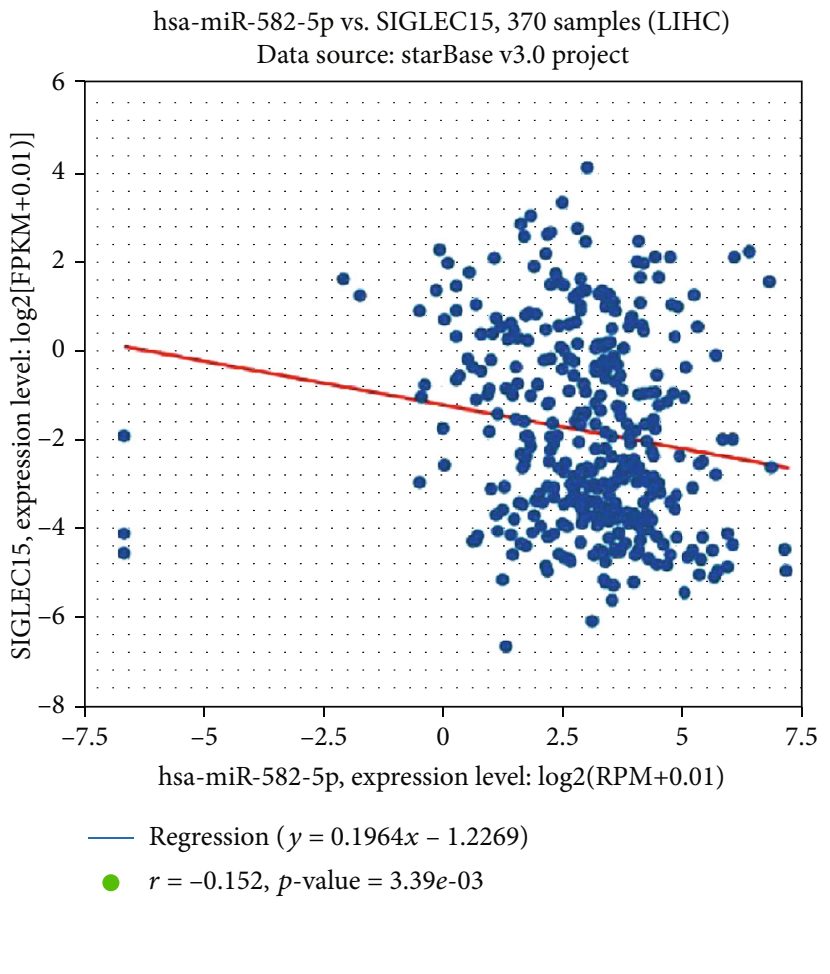

(d)

Figure 2: Continued. 


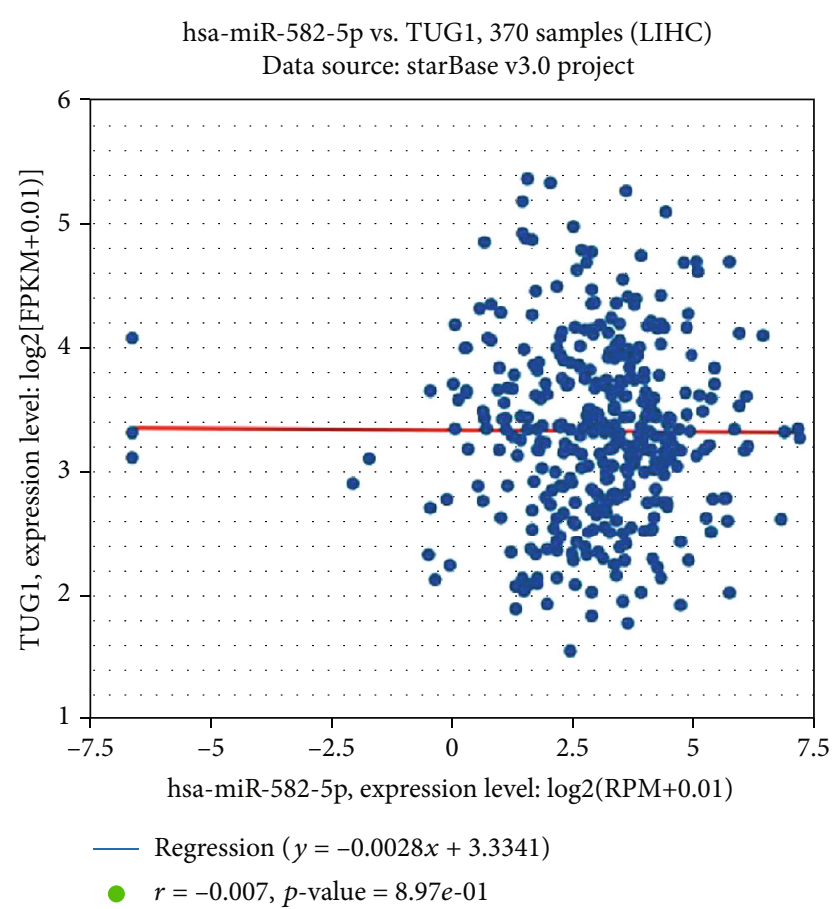

(e)

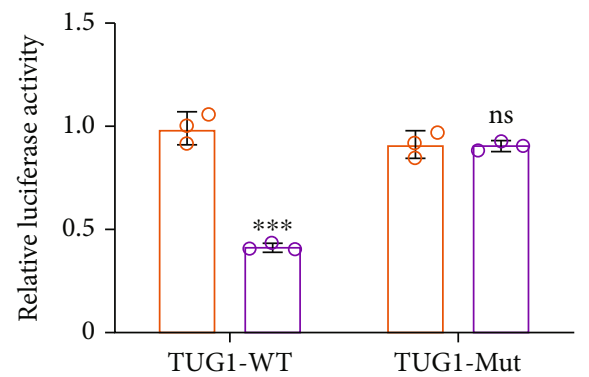

O mi-NC

O hsa-miR-582-5p

(g)

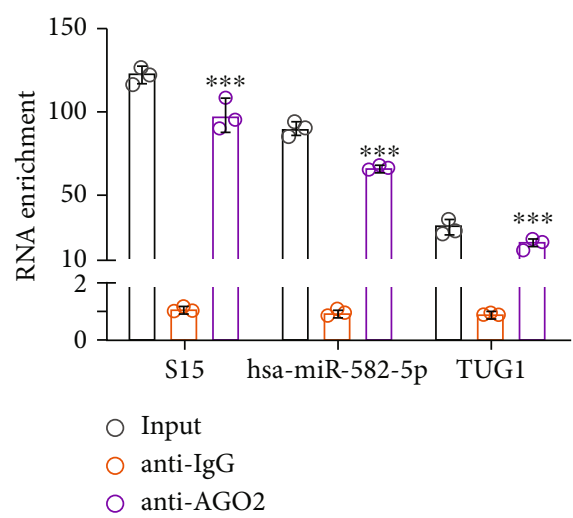

(i)
Has-miR-582-5p: $3^{\prime}$ UCAUUGACCAACUUGUUGACAUU 5'

TUG1-Mut: 5' UGCGG----AAGCACGACCAGCACU 3'

S-15-WT: 5' AACAUCCAUUUCAGCACUGUAAA 3'

|| || | |

Has-miR-582-5p: 3' UCAUUGACCAACUUGUUGACAUU 5'

S15-Mut: 5' AACAUCCAUUUCAGCGACAGUGA 3'

(f)

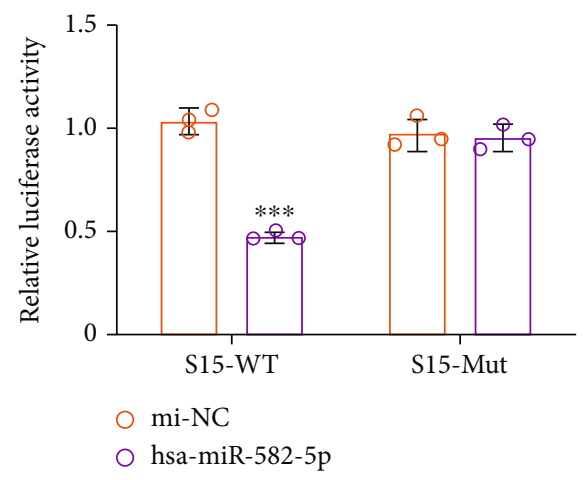

(h)

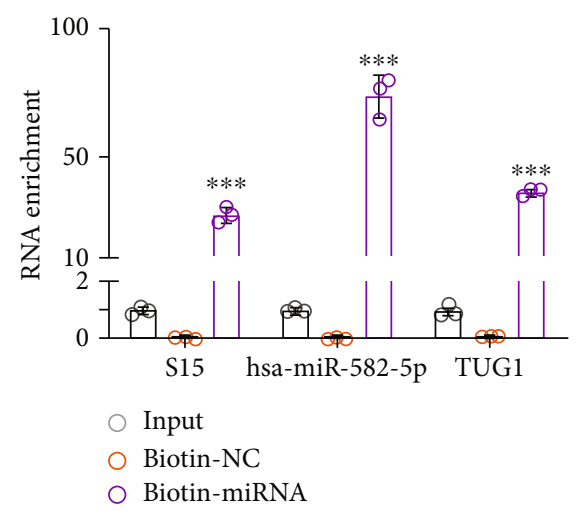

(j)

Figure 2: Continued. 


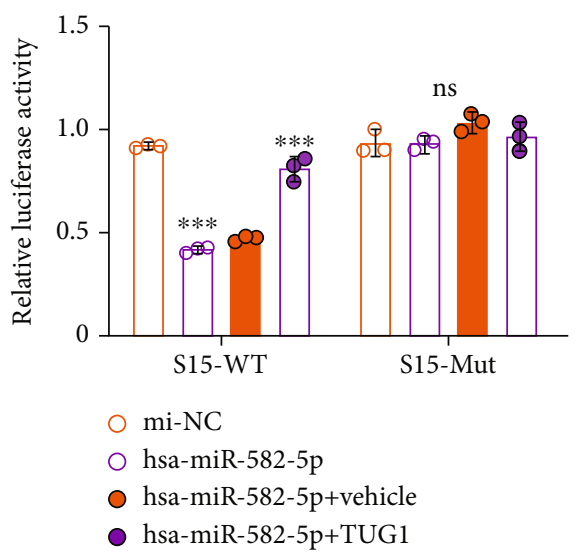

(k)

FIGURE 2: hsa-miR-582-5p directly binds to TUG1 and $3^{\prime}$-UTR of Siglec-15. (a) The intersection of predicted miRNAs targeting TUG1 and Siglec-15. Relative luciferase activity of dual-luciferase assay in miRNAs screen targeting (b) Siglec-15 and (c) TUG1. The coexpression relationship between hsa-miR-582-5p and (d) Siglec-15 or (e) TUG1 in clinical data from TCGA database. (f) The predicted binding sequence between hsa-miR-582-5p and TUG1 or Siglec-15. Relative luciferase activity of dual-luciferase assay in hsa-miR-582-5p targeting (g) Siglec-15 and (h) TUG1. (i) RIP assay in Hep3B cells coprecipitated with AGO2 protein. (j) RNA pull-down assay in Hep3B cells coprecipitated with biotin-labeled hsa-miR-582-5p. (k) Relative luciferase activity of dual-luciferase assay in hsa-miR-582-5p targeting Siglec-15 with TUG1 regulation. The data are presented as the means $\pm \mathrm{SD}, n=3$ replicates in $(\mathrm{b}, \mathrm{c}$, and $\mathrm{g}-\mathrm{k}) .{ }^{*} P<0.05,{ }^{* *} P<$ $0.01,{ }^{* * *} P<0.005$. S15: Siglec- 15 .

reduction in HCC patients compared to healthy individuals $(P=0.028)$ (Figure 3(a)) [15]. We also measured the expression of hsa-miR-582-5p in 6 HCC cell lines, LO-2 cells, and $\mathrm{HH}$ cells. We found a general decrease in hsa-miR-582-5p expression in the HCC cell lines compared to LO-2 and $\mathrm{HH}$ cells (Figure 3(b)). Next, we explored the effects of hsa-miR-582-5p on Siglec-15 and TUG1 expression. qPCR and western blot analyses showed that transfection with hsa-miR-582-5p inhibited Siglec-15 abundance, while the inhibitor of hsa-miR-582-5p increased Siglec-15 expression, indicating that hsa-miR-582-5p negatively regulates Siglec15 expression (Figures 3(c)-3(f)). However, TUG1 expression displayed no significant changes with the variable hsamiR-582-5p level (Figures 3(c) and 3(d)). We found that the artificially controlled TUG1 level in Hep3B and HUH7 cells failed to affect hsa-miR-582-5p expression (Figures 3(g) and 3(h)). The noninterference performances between TUG1 and hsa-miR-582-5p were in accordance with the ceRNA mechanism.

To further confirm the necessity of hsa-miR-582-5p in TUG1-induced Siglec-15 regulation, we used hsa-miR-582$5 p$ knockout HUH7 and Hep3B cell lines (HUH7-miRKO and Hep3B-miRKO, respectively). Notably, the expression of Siglec-15 remained unchanged with the variable TUG1 level in hsa-miR-582-5p knockout cell lines at either the mRNA or protein level, indicating that Siglec-15 expression is not controlled by TUG1 when hsa-miR-582-5p is knocked out (Figures 3(i)-3(l)). These results suggested that hsamiR-582-5p is necessary for TUG1-regulated Siglec-15 expression.

In addition, we evaluated the role of hsa-miR-582-5p in the immune response in HCC cells. hsa-miR-582-5p overexpression increased the luciferase activity in the RGA assay, but the inhibitor of hsa-miR-582-5p reduced the luciferase activity (Figures 3(m) and 3(n)). Similarly, higher levels of cytotoxicity as well as IFN- $\gamma$ and IL- 2 concentrations were found in HCC cells overexpressing hsa-miR-582-5p, and the opposite effect was found when treated with the inhibitor of hsa-miR-582-5p (Figures 3(o)-3(t)). These findings demonstrated that hsa-miR-582-5p impacts the $\mathrm{T}$ cell-induced immune response in $\mathrm{HCC}$ cells by regulating Siglec-15 expression.

3.4. siRNAs Screen for Developing RNAi Drugs. Our initial aim was to identify a target for siRNA drugs in HCC therapy by inhibiting Siglec-15 abundance. Except for the function of TUG1 in Siglec-15 regulation identified in our study, other studies have demonstrated that TUG1 is an oncogene that promotes cancer development and increases tumor malignancy, including tumor cell survival, proliferation, migration, and invasion, in cancers such as HCC [10-13]. Thus, TUG1 has a dual role in cancer development as it promotes the anti-immune response and oncogenicity, indicating that it is an ideal target for siRNA drug development. Once a siRNA knocks down TUG1 in HCC, different mechanisms will be working to eliminate the tumor by suppressing its progression and improving the immune response. Therefore, we began to develop a si-TUG1 sequence with high knockdown efficiency.

First, we designed a high-throughput method using HUH7 and Hep3B cells stably expressing TUG1-IRESEGFP genes using the EGFP signaling to replace the TUG1 abundance. Once siRNA candidates were capable of TUG1 knockdown, EGFP expression was inhibited, allowing siRNAs to be screened by flow cytometry (Figure 4(a)). Second, the target region for siRNAs was predicted to be the conserved region in the TUG1 sequence, thereby avoiding efficacy loss due to mutation. We aligned the TUG1 


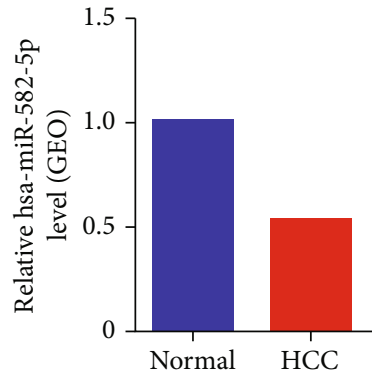

(a)

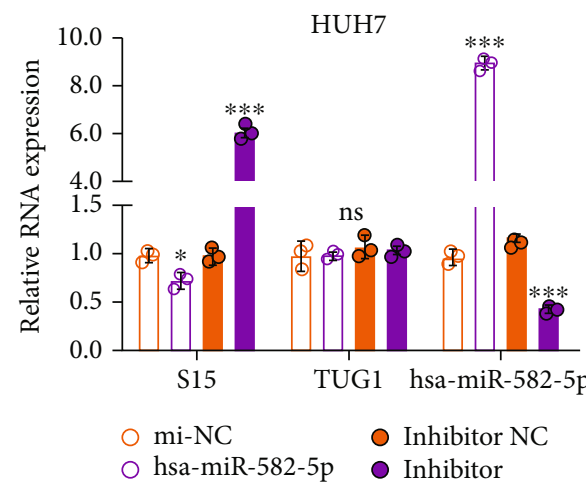

(c)

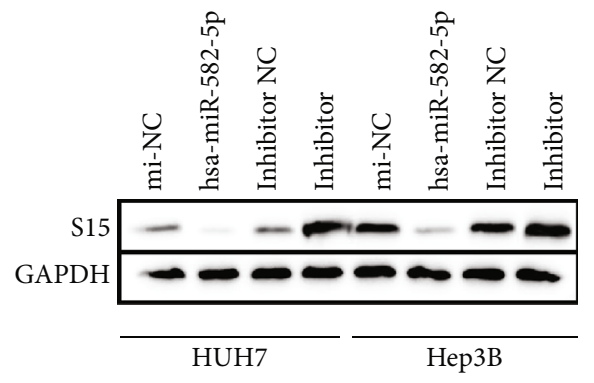

(e)

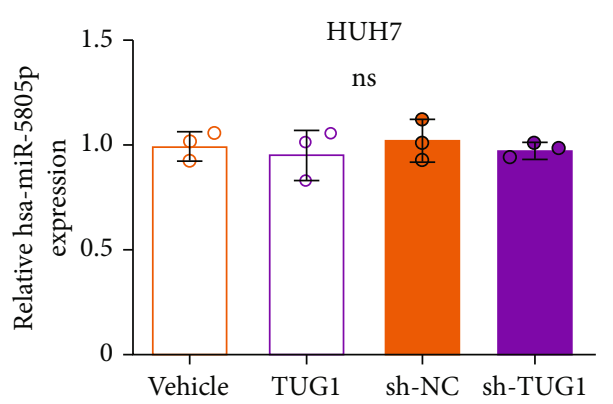

(g)

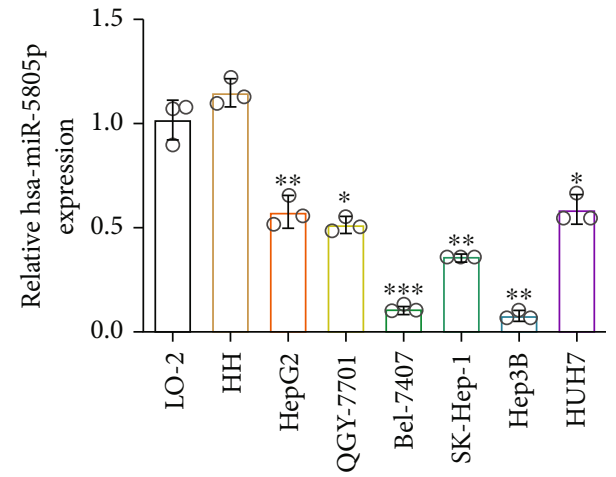

(b)

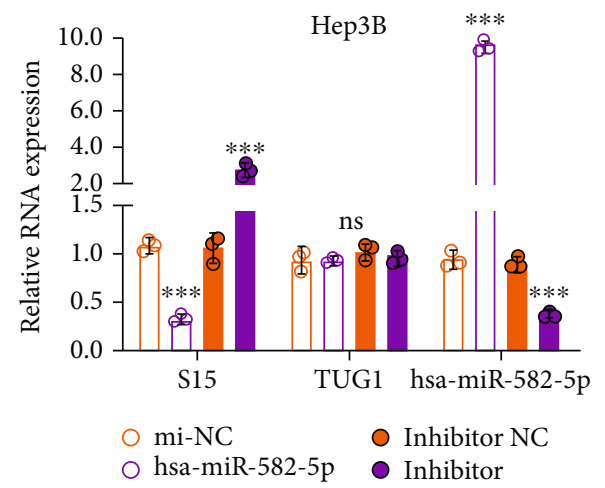

(d)

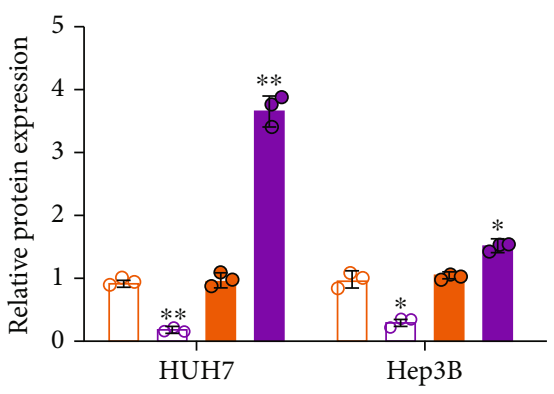

$\circ$ mi-NC O Inhibitor NC

○ hsa-miR-582-5p ○ Inhibitor

(f)

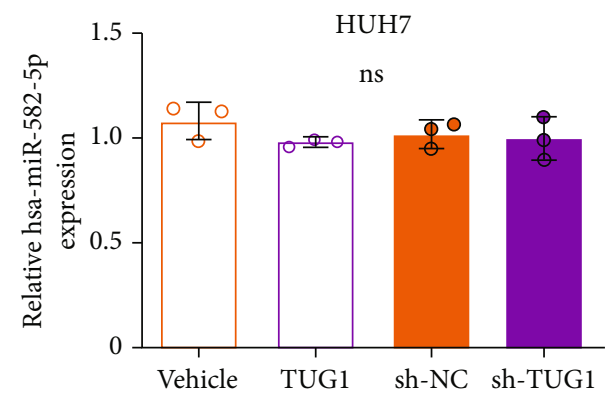

(h)

FIgURE 3: Continued. 


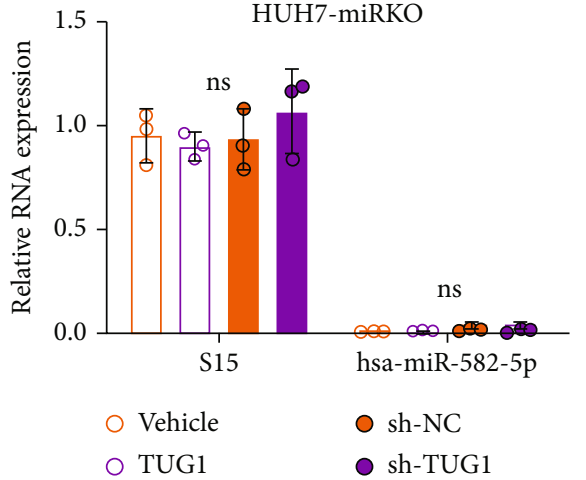

(i)

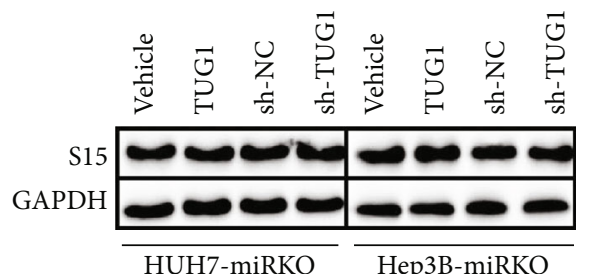

Hep3B-miRKO

(k)

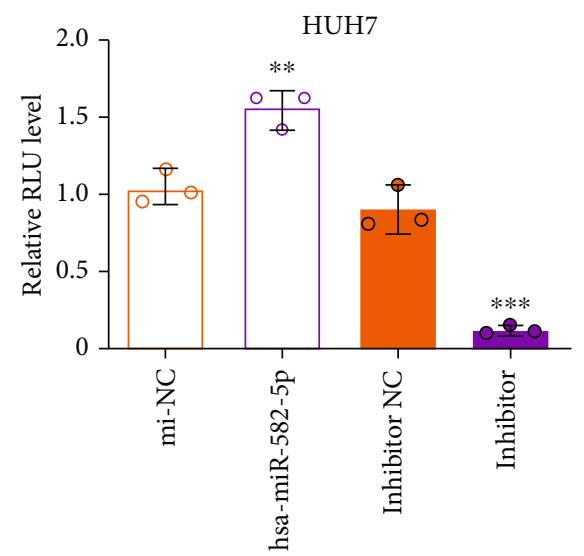

(m)

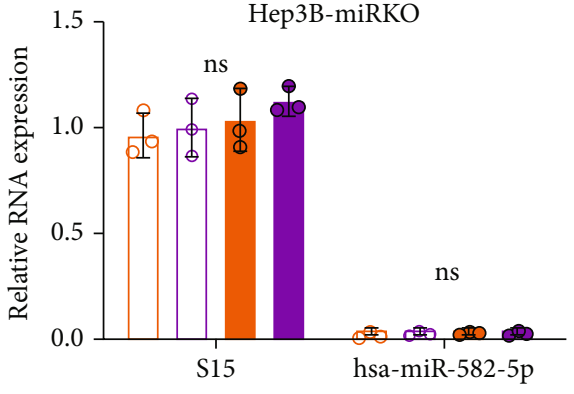

○ Vehicle $\quad$ sh-NC

○ TUG1

(j)

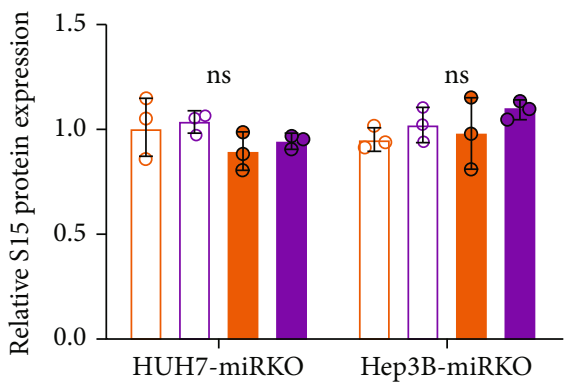

Vehicle $\quad$ sh-NC

○ TUG1 $\quad$ sh-TUG1

(1)

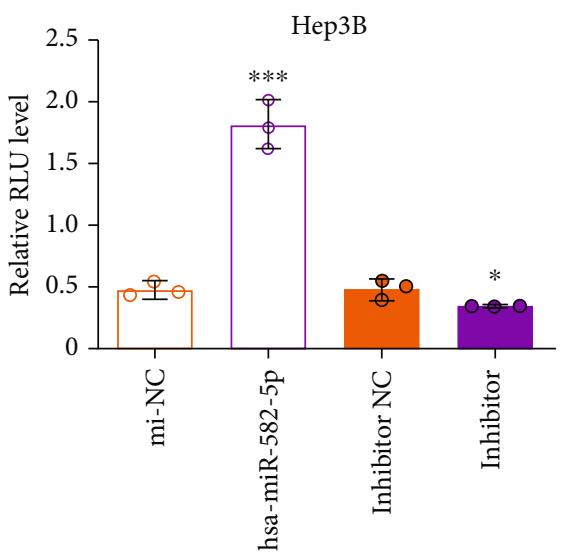

(n)

Figure 3: Continued. 


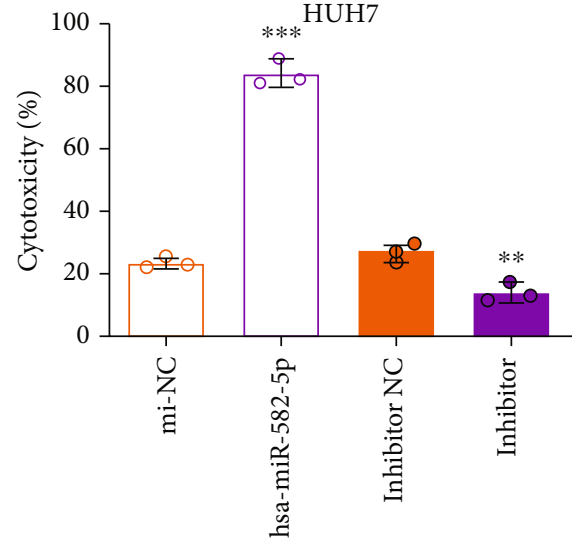

(o)

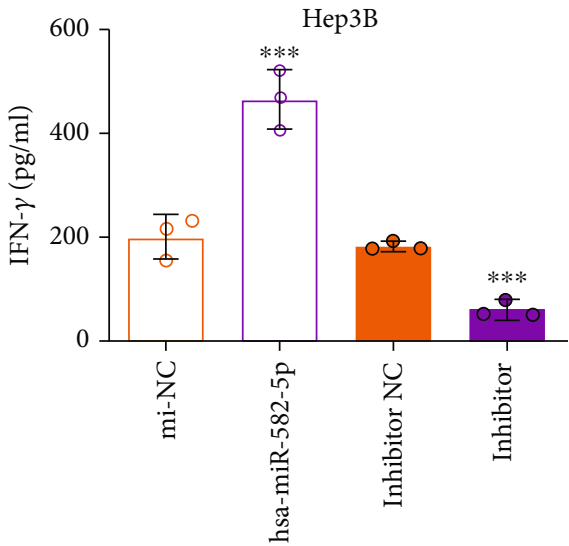

(q)

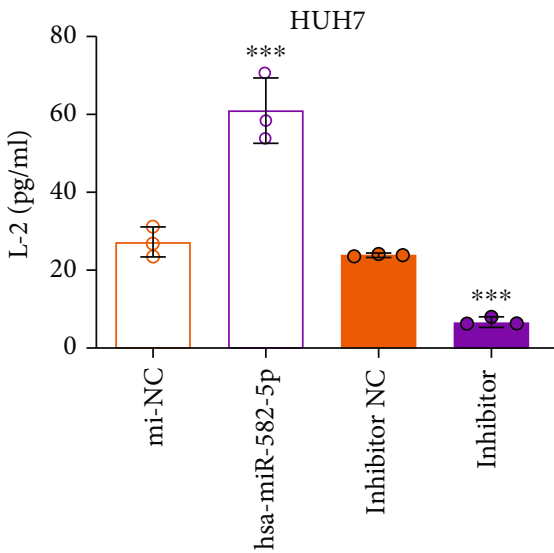

(s)

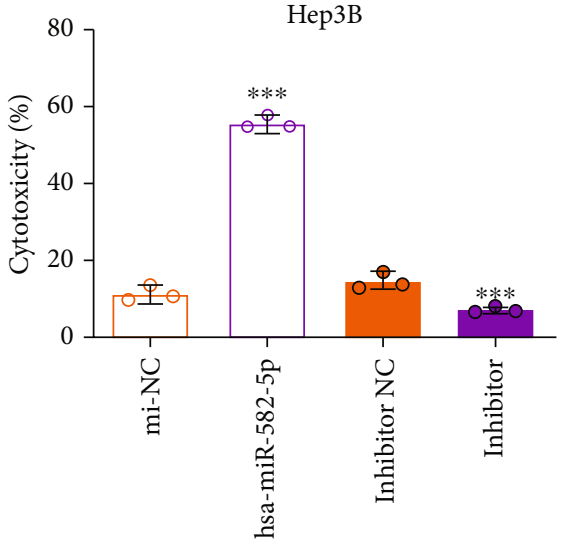

(p)

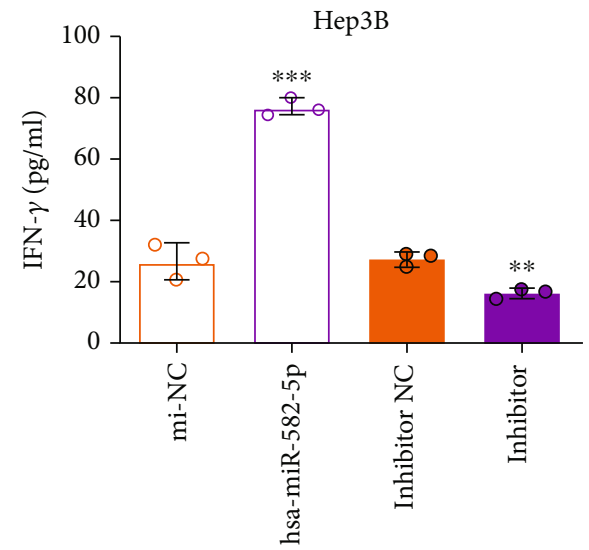

$(\mathrm{r})$

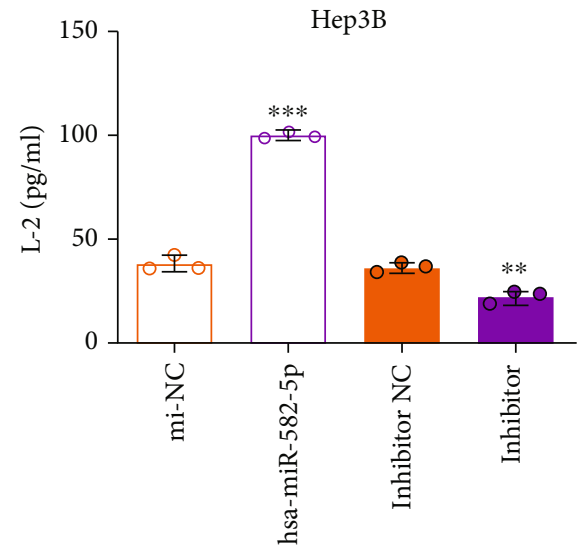

(t)

FIGURE 3: hsa-miR-582-5p negatively regulates Siglec-15 level and its induced immunosuppression. (a) hsa-miR-582-5p expression in HCC patients and normal person from GEO analysis (GPL8179). (b) hsa-miR-582-5p in different HCC cell lines (HepG2, QGY-7701, Bel-7407, SK-Hep-1, Hep3B, and HUH7), human normal hepatic cell line LO-2, and primary human hepatocytes HH. (c, f) TUG1 expression, Siglec15 mRNA expression, and protein expression in HUH7 and Hep3B cells treated with hsa-miR-582-5p mimics or inhibitor. hsa-miR-582-5p expression in (g) HUH7 and (h) Hep3B cells treated TUG1 overexpression or knockdown. (i, j) hsa-miR-582-5p expression, (i, j) Siglec-15 mRNA, and $(\mathrm{k}, \mathrm{l})$ protein expression in HUH7-miRKO and Hep3B-miRKO cells treated TUG1 overexpression or knockdown. (m, $\mathrm{n}$ ) Relative luciferase activity of Jurkat-RGA in HUH7 and Hep3B cells treated with hsa-miR-582-5p mimics or inhibitor. (o, p) T cellinduced cytotoxicity cocultured with HUH7 and Hep3B cells treated with hsa-miR-582-5p mimics or inhibitor. (q, r) IFN- $\gamma$ and (s, $t$ ) IL-2 secreted by $\mathrm{T}$ cells from (o, p). The data are presented as the means $\pm \mathrm{SD}, n=3$ replicates in $(\mathrm{b}-\mathrm{t}) .{ }^{*} P<0.05,{ }^{* *} P<0.01,{ }^{* * *} P<$ 0.005. S15: Siglec-15. 


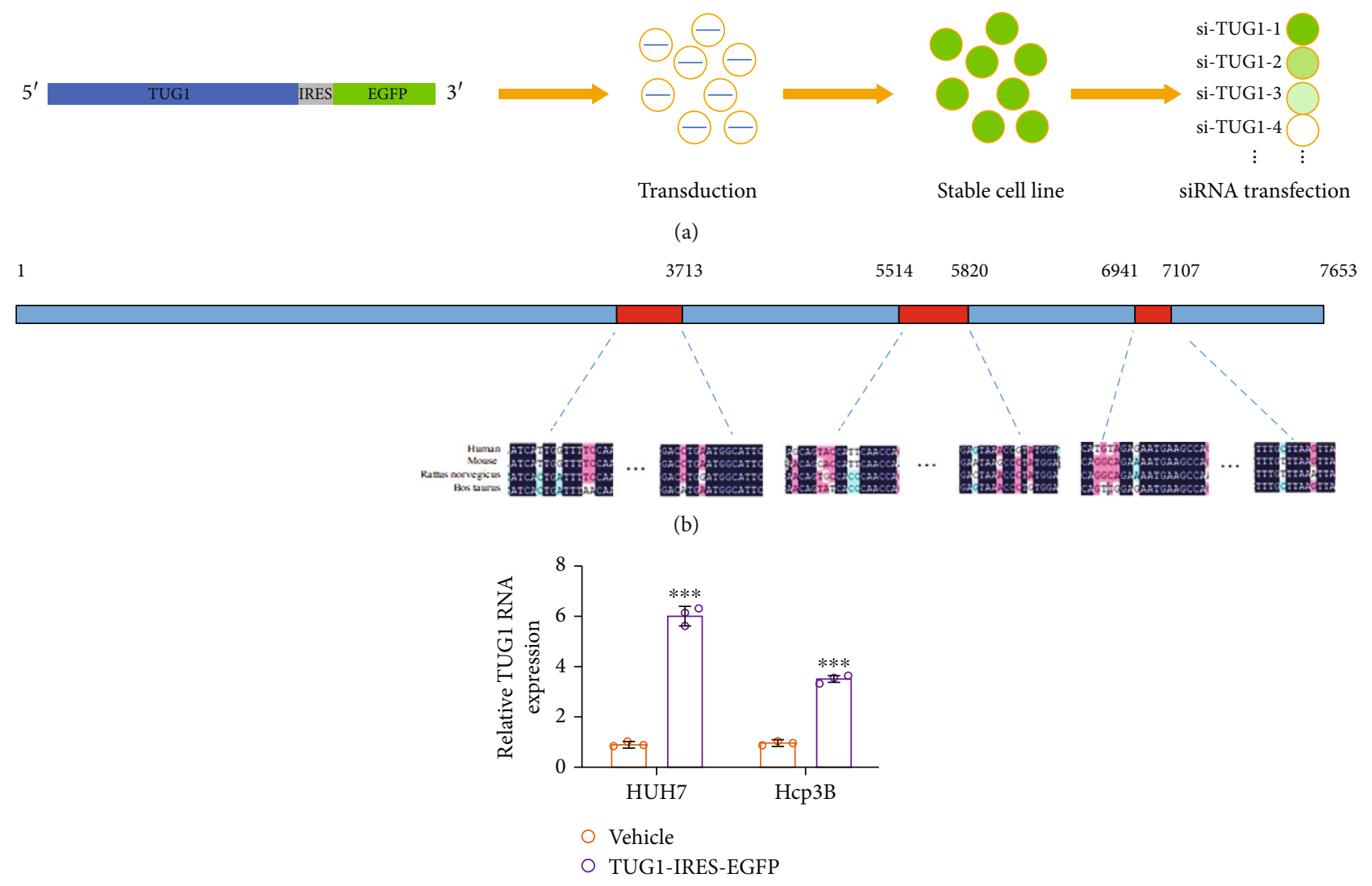

(c)
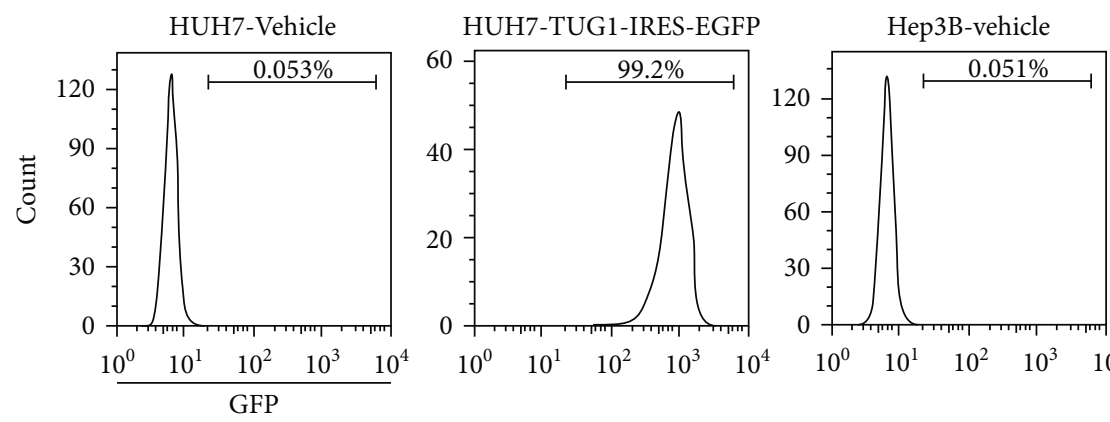

Hep3B-TUG1-IRES-EGFP

(d)

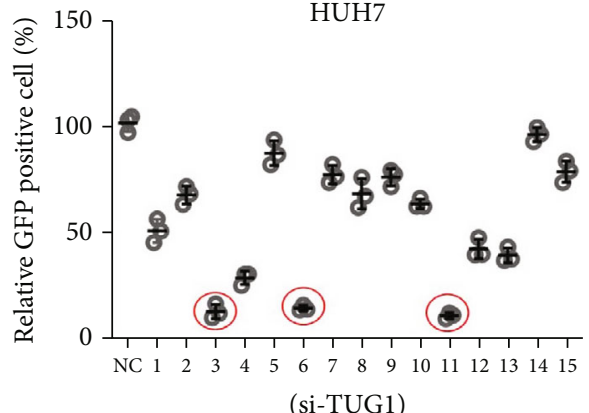

(e)

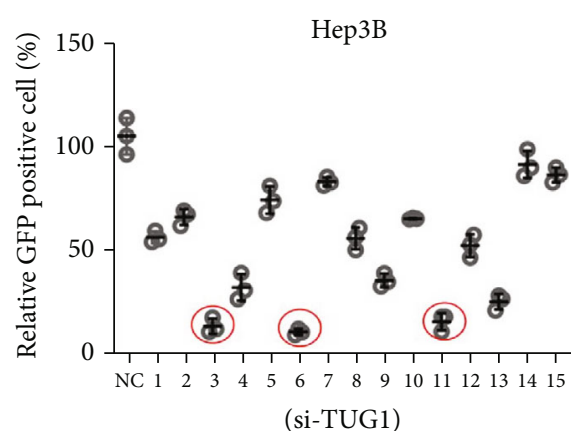

(f)

Figure 4: Continued. 


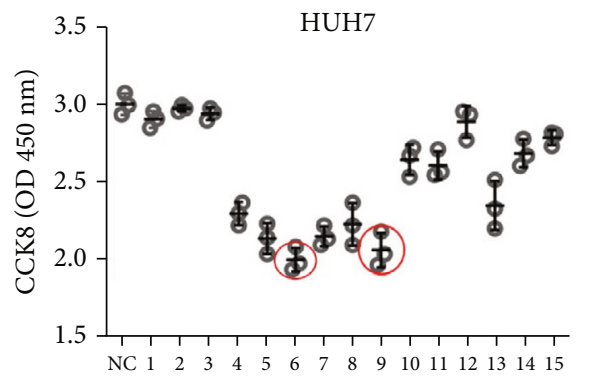

(si-TUG1)

(g)

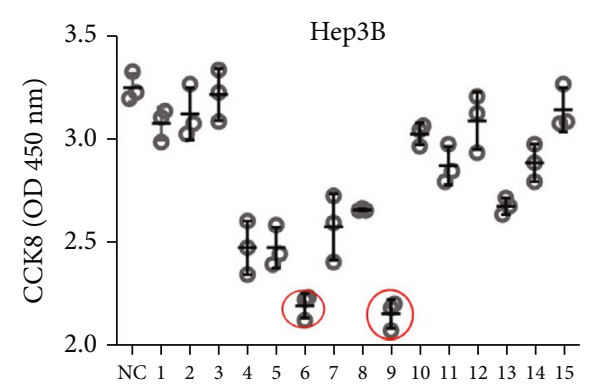

(si-TUG1)

(h)

FIgURE 4: The screen of siRNAs targeting TUG1. (a) Schematic diagram of the siRNA screen method and cell line construction. (b) Schematic diagram of the TUG1 conserved region. (c) TUG1 expression in HUH7-TUG1-IRES-EGFP and Hep3B-TUG1-IRES-EGFP cells. (d) Flow cytometry results of EGFP positive rate in HUH7-TUG1-IRES-EGFP and Hep3B-TUG1-IRES-EGFP cells. The screen of siRNAs targeting TUG1 in (e) HUH7-TUG1-IRES-EGFP and (f) Hep3B-TUG1-IRES-EGFP (F) cells by flow cytometry. The screen of siRNAs targeting TUG1 in (g) HUH7 and (h) Hep3B cells by CCK8. The data are presented as the means \pm SD, $n=3$ replicates in (c and e-h). ${ }^{*} P<0.05,{ }^{* *} P<0.01,{ }^{* * *} P<0.005$. S15: Siglec- 15 .

sequences in humans, mice, rats, and cattle from the National Center of Biotechnology Information (NCBI). The regions of $3453-3713 \mathrm{bp}, 5514-5820 \mathrm{bp}$, and 6941 $7107 \mathrm{bp}$ in the human TUG1 sequence were conserved regions with high homology among different species (Figure 4(b)). Fifteen siRNAs were designed according to the conserved regions by siDirect (http://sidirect2.rnai.jp/) (Table 1). The cell lines stably expressing TUG1-IRESEGFP were confirmed by qPCR (measuring TUG1 expression) and flow cytometry (measuring EGFP expression) (Figures 4(c) and 4(d)). After the siRNA screen, we found that si-TUG1-3, si-TUG1-6, and si-TUG1-11 displayed the best level of TUG1 inhibition in both HUH7 and Hep3B cells (Figures 4(e) and 4(f)).

Because many studies have indicated that TUG1 inhibits tumor cell proliferation in HCC $[10,16]$, we used a CCK-8 assay to screen the performance of the 15 siRNAs $72 \mathrm{~h}$ after transfecting them into wild-type HUH7 and Hep3B cells. The results indicated that si-TUG1-6 and si-TUG1-9 inhibited cell proliferation better than the other siRNAs (Figures 4(g) and 4(h)). Because si-TUG1-6 had the strongest effect on TUG1 downregulation and cell proliferation inhibition in HCC cells, we used it for subsequent experiments.

3.5. si-TUG1-6 Suppresses Tumor Development by Reducing Tumor Cell Proliferation and Increasing the Immune Response. To verify the function of si-TUG1-6, we first transfected it into HCC cells overexpressing TUG1-IRESEGFP. The immunofluorescence results showed a remarkable reduction in EGFP expression (Figures 5(a) and 5(b)). Next, we knocked down TUG1 expression in wild-type HUH7 and Hep3B cells with si-TUG1-6, resulting in a nearly $80 \%$ reduction. Moreover, the Siglec-15 level was reduced by $70-80 \%$ in cells treated with si-TUG1-6 (Figures 5(d)-5(f)). These results suggested that si-TUG1-6 is highly efficient in suppressing TUG1 levels in HCC cells.

Subsequently, we evaluated the effects of si-TUG1-6 on the immune response in HUH7 and Hep3B cells. si-TUG16 caused the luciferase activity in the Jurkat-RGA assay to significantly increase, which was consistent with the $\mathrm{T}$ cellinduced cytotoxicity and cytokine release (Figures 5(g)$5(\mathrm{n}))$.

In addition, we also evaluated the function of si-TUG1-6 in tumor oncogenicity in HCC cells. We found that siTUG1-6 overexpression severely inhibited the proliferation of HUH7 and Hep3B cells (Figures 5(o)-5(r)). The apoptosis of HUH7 and Hep3B cells was also increased after treatment with si-TUG1-6 (Figures 5(s) and 5(t)), consistent with upregulated $\mathrm{C}$-caspase 3 and $\mathrm{Bcl}-2$ protein levels as well as reduced caspase 3 protein levels (Figures $5(\mathrm{u})$ and $5(\mathrm{v})$ ). The migration and invasion abilities of HUH7 and Hep3B cells were also measured, and the results showed that siTUG1-6 effectively repressed malignant tumor behavior (Figures 5(w)-5(z)).

Together, these findings suggested that si-TUG1-6 is an efficient antitumor inhibitor by suppressing tumor progression and improving the immune response via downregulating Siglec-15 in HCC cells in vitro.

3.6. si-TUG1-6 Exhibits a Synergistic Antitumor Effect in HCC In Vivo. A xenograft model in NCG mice was used to evaluate the antitumor efficacy of si-TUG1-6 in HCC with the following groups (Figure 6(a)): WNP, mice were injected with wild-type Hep3B cells (WT, W), si-NC (N), and PBMCs (P); KNP, mice were injected with Hep3B-miRKO cells (miRKO, K), si-NC (N), and PBMCs $(\mathrm{P})$; KTP, mice were injected with Hep3B-miRKO cells (miRKO, K), siTUG1-6 (T), and PBMCs (P); WTP, mice were injected with wild-type Hep3B cells (WT, W), si-TUG1-6 (T), and PBMCs (P); WNN, a control group to reflect original tumor growth in PBMCs; and KNN, a control group to demonstrate the effect of hsa-miR-582-5p knockout on tumor growth in PBMCs. The KTP group was designed to eliminate the influence of si-TUG1-6 on Siglec-15 in tumor cells as TMG2 failed to disturb Siglec-15 expression with hsa-miR-582-5p knockout in vitro (Figures 3(i)-3(1)). Therefore, the change in tumor growth in the KTP group was only attributed to the tumor cell oncogenicity induced by si-TUG1-6 compared to the WNP and KNP groups. WTP was a group 
TABLE 1: siRNA sequences.

\begin{tabular}{|c|c|c|}
\hline No. & $\begin{array}{c}\text { Target sequence } \\
21 \text { nt target }+2 \text { nt overhang }\end{array}$ & $\begin{array}{l}\text { RNA oligo sequences } \\
\text { 21nt guide }\left(5^{\prime} \rightarrow 3^{\prime}\right) \\
21 \mathrm{nt} \text { passenger }\left(5^{\prime} \rightarrow 3^{\prime}\right)\end{array}$ \\
\hline \multicolumn{3}{|c|}{3453 bp-3713 bp } \\
\hline si-TUG1-1 & GGCCTGAATCCTGCTACAACTAT & $\begin{array}{l}\text { AGUUGUAGCAGGAUUCAGGCC } \\
\text { CCUGAAUCCUGCUACAACUAU }\end{array}$ \\
\hline si-TUG1-2 & TGCTACAACTATCTTCCTTTACC & $\begin{array}{l}\text { UAAAGGAAGAUAGUUGUAGCA } \\
\text { CUACAACUAUCUUCCUUUACC }\end{array}$ \\
\hline si-TUG1-3 & TACAACTATCTTCСТTTACСACC & $\begin{array}{l}\text { UGGUAAAGGAAGAUAGUUGUA } \\
\text { CAACUAUCUUCCUUUACCACC }\end{array}$ \\
\hline si-TUG1-4 & TTCCTTACAACACCTTGAACTCT & $\begin{array}{l}\text { AGUUCAAGGUGUUGUAAGGAA } \\
\text { CCUUACAACACCUUGAACUCU }\end{array}$ \\
\hline \multicolumn{3}{|c|}{5514 bp-5820 bp } \\
\hline si-TUG1-5 & GCCTTGACTTGCTTGTAAGATGA & $\begin{array}{l}\text { AUCUUACAAGCAAGUCAAGGC } \\
\text { CUUGACUUGCUUGUAAGAUGA }\end{array}$ \\
\hline si-TUG1-6 & ACGACTTGATTACCAAAGAAAGT & $\begin{array}{l}\text { UUUCUUUGGUAAUCAAGUCGU } \\
\text { GACUUGAUUACCAAAGAAAGU }\end{array}$ \\
\hline si-TUG1-7 & GACTTGATTACCAAAGAAAGTAG & $\begin{array}{l}\text { ACUUUCUUUGGUAAUCAAGUC } \\
\text { CUUGAUUACCAAAGAAAGUAG }\end{array}$ \\
\hline si-TUG1-8 & TAGCATAGACTCCTAAACAGAAC & $\begin{array}{l}\text { UCUGUUUAGGAGUCUAUGCUA } \\
\text { GCAUAGACUCCUAAACAGAAC }\end{array}$ \\
\hline si-TUG1-9 & AGCATAGACTCCTAAACAGAACC & $\begin{array}{l}\text { UUCUGUUUAGGAGUCUAUGCU } \\
\text { CAUAGACUCCUAAACAGAACC }\end{array}$ \\
\hline si-TUG1-10 & CTGTAAGATCAGAAAACTGTATC & $\begin{array}{l}\text { UACAGUUUUCUGAUCUUACAG } \\
\text { GUAAGAUCAGAAAACUGUAUC }\end{array}$ \\
\hline \multicolumn{3}{|c|}{$6941 b p-7107 b p$} \\
\hline si-TUG1-11 & CTGGACTTTTCAGTTATGTGAAC & $\begin{array}{l}\text { UCACAUAACUGAAAAGUCCAG } \\
\text { GGACUUUUCAGUUAUGUGAAC }\end{array}$ \\
\hline si-TUG1-12 & TGGACTTTTCAGTTATGTGAACC & $\begin{array}{l}\text { UUCACAUAACUGAAAAGUCCA } \\
\text { GACUUUUCAGUUAUGUGAACC }\end{array}$ \\
\hline si-TUG1-13 & TTCAGTTATGTGAACCAATAAAT & $\begin{array}{l}\text { UUAUUGGUUCACAUAACUGAA } \\
\text { CAGUUAUGUGAACCAAUAAAU }\end{array}$ \\
\hline si-TUG1-14 & CAGTTATGTGAACCAATAAATAC & $\begin{array}{l}\text { AUUUAUUGGUUCACAUAACUG } \\
\text { GUUAUGUGAACCAAUAAAUAC }\end{array}$ \\
\hline si-TUG1-15 & AACCAATAAATACCCTTTTTTGC & $\begin{array}{l}\text { AAAAAAGGGUAUUUAUUGGUU } \\
\text { CCAAUAAAUACCCUUUUUUGC }\end{array}$ \\
\hline
\end{tabular}

designed to reflect the synergetic effects on tumor growth induced by si-TUG1-6.

There were no significant differences between the WNN and KNN groups, which indicated that hsa-miR-582-5p knockout did not affect tumor growth in HCC (Figures 6(a)-6(c)). The results of the KTP group, which showed reduced tumor growth compared to the WNN and KNN groups, demonstrated that this reduction was due to the inhibition of tumor progression induced by si-TUG1-6 and that hsa-miR-582-5p knockout did not affect tumor growth (Figures 6(a)-6(c)). More importantly, the WTP group showed more suppression of tumor growth than the KTP group, indicating that the increased antitumor activity was attributed to si-TUG1-6-induced Siglec-15 downregula- tion (Figures 6(a)-6(c)). These results revealed that siTUG1-6 displays a synergetic antitumor effect by inhibiting tumor progression and increasing immune activity.

In addition, we also measured the expression of Siglec-15 and TUG1 in tumors from different groups. The results showed no change in Siglec-15 levels in all groups, except the WTP group (Figures 6(d) and 6(e)), which showed decreased Siglec-15 expression (Figures 6(d)-6(f)). TUG1 expression was only reduced in tumors treated with siTUG1-6 (Figure 6(f)). Moreover, hsa-miR-582-5p level was not expressed in the miRKO group (Figure 6(f)). These results support the conclusion drawn from Figures 6(a)-6(c).

To further analyze the influence of Siglec-15 levels on T cell cytotoxic activity, we isolated PBMCs from KTP and 


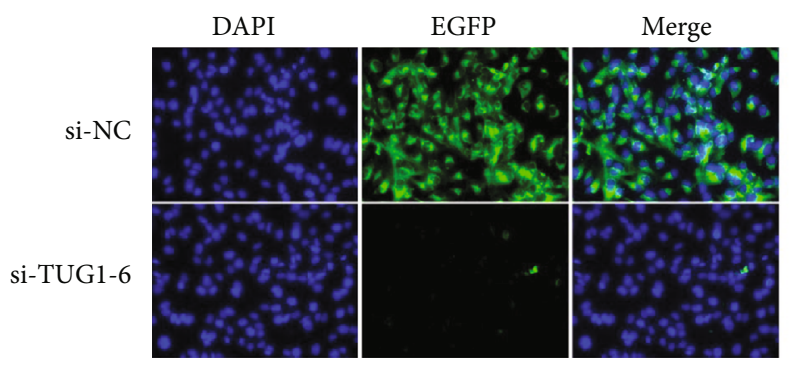

HUH7-TUG1-IRES-EGFP

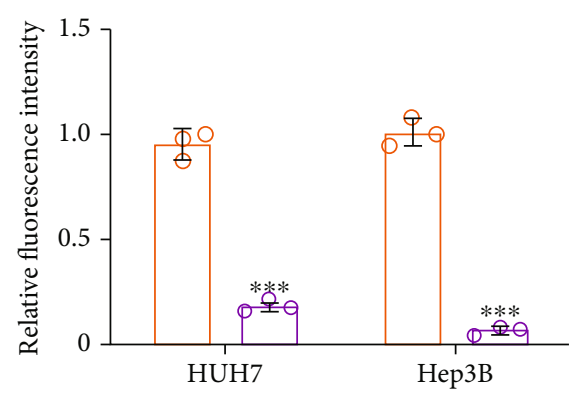

O si-NC

si-TUG1-6

(b)

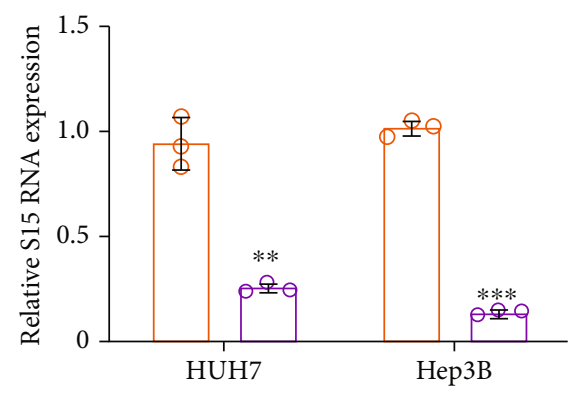

$\mathrm{O}$ si-NC

o si-TUG1-6

(d)

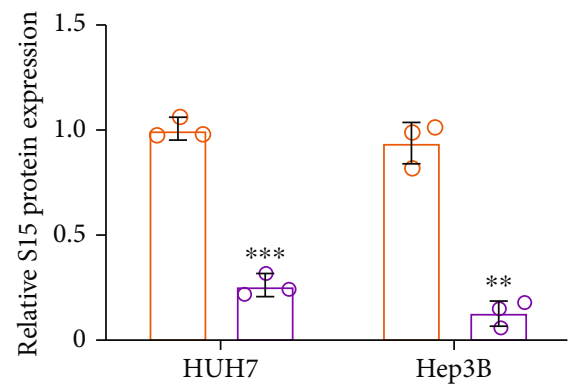

si-NC

O si-TUG1-6

(f)

(a)

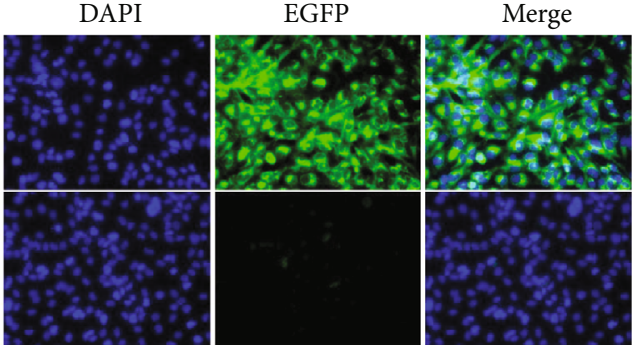

Hep3B-TUG1-IRES-EGFP

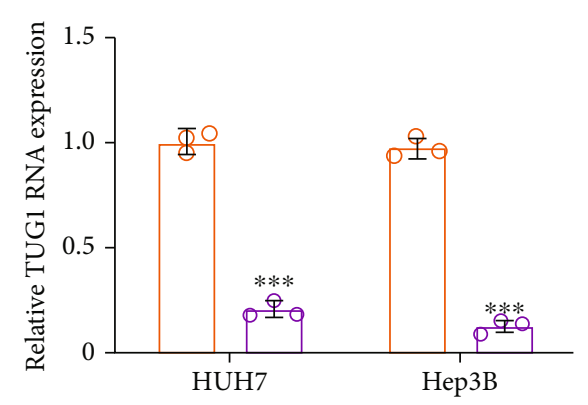

si-NC

si-TUG1-6

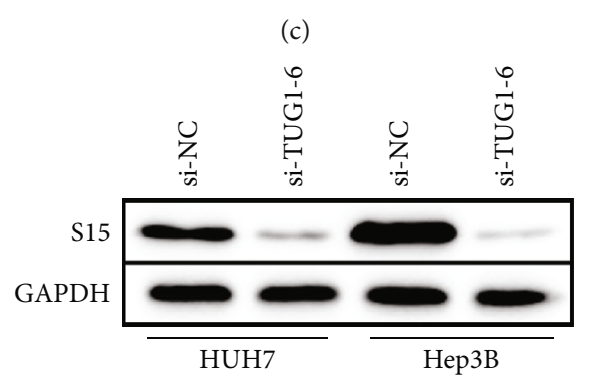

(e)

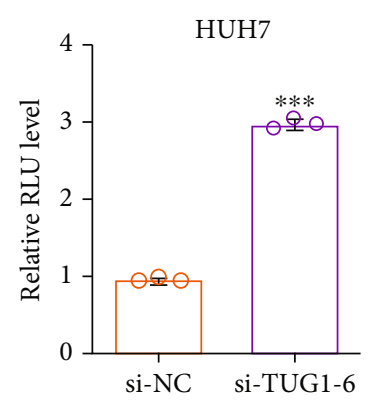

(g)

Figure 5: Continued. 


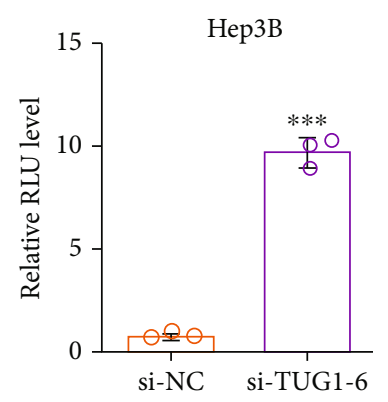

(h)

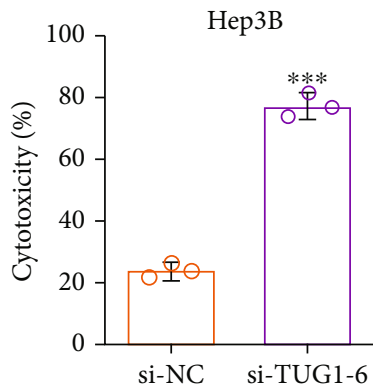

(j)

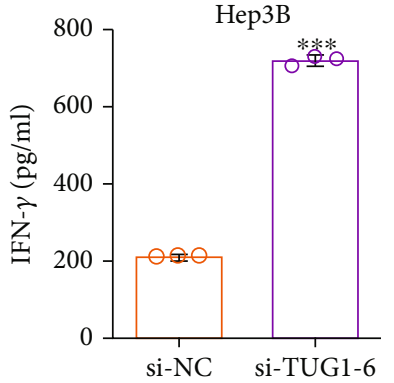

(l)
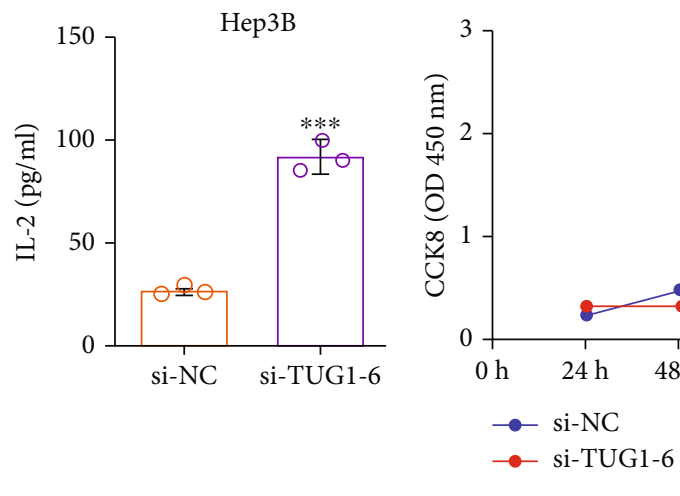

(n)

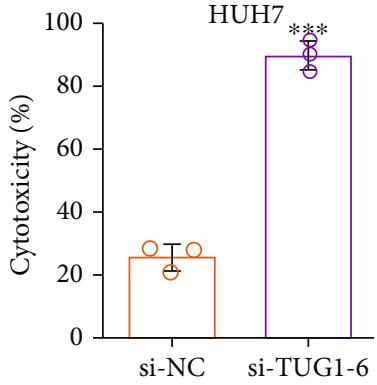

(i)

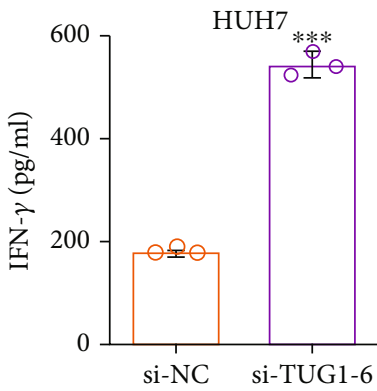

(k)

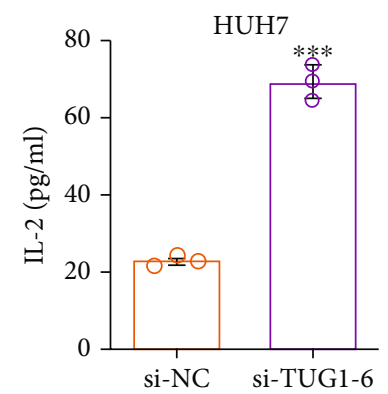

(m)

HUH7

(o)

Figure 5: Continued. 


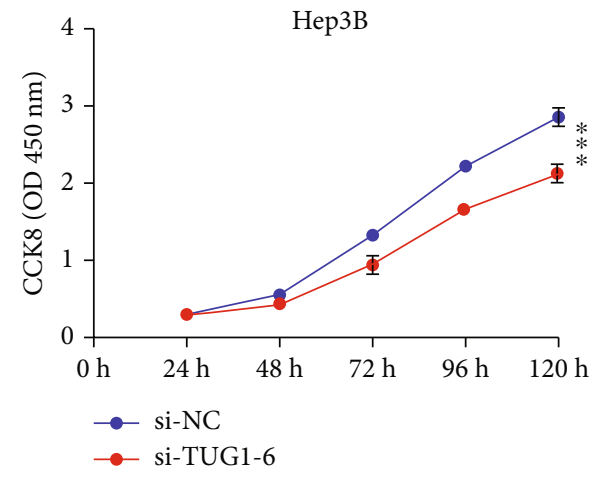

(p)

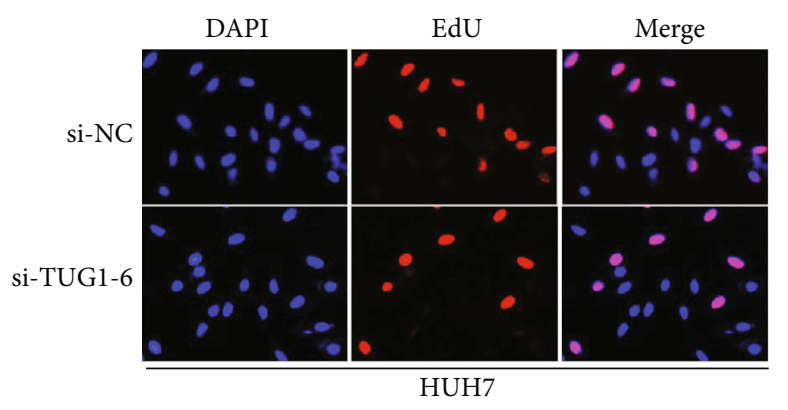

(q)

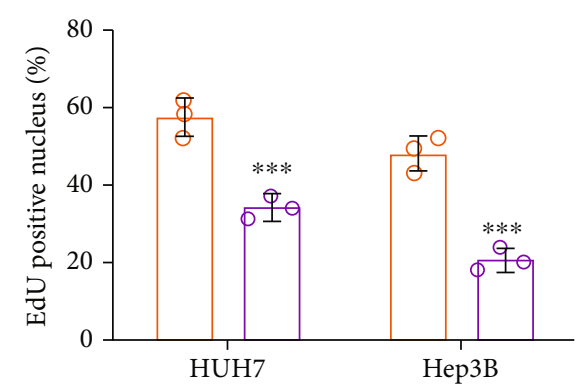

si-NC

o si-TUG1-6

(r)

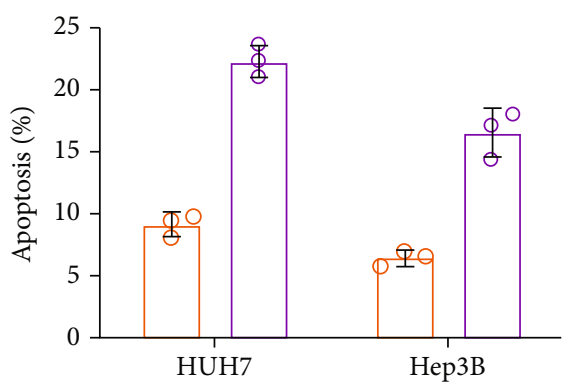

O si-NC

O si-TUG1-6
DAPI
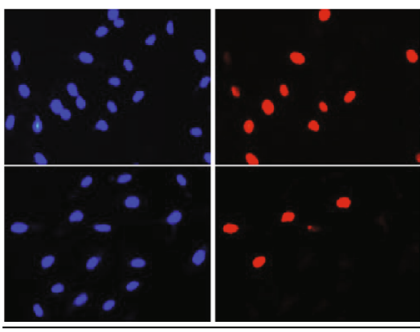

Hep3B

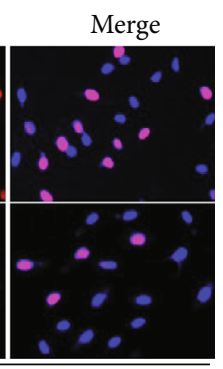

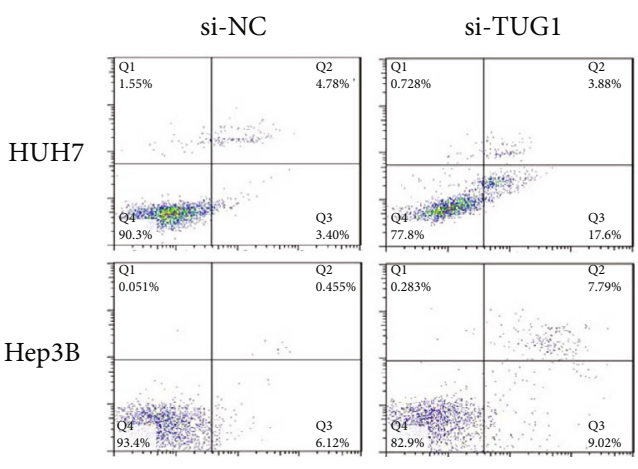

(s)

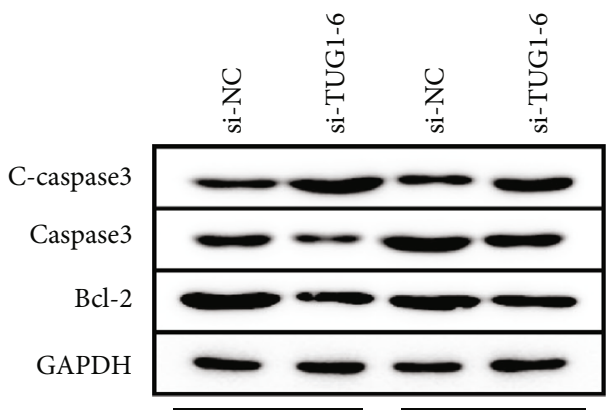

HUH7

Hep3B

(u)

FIgURe 5: Continued. 


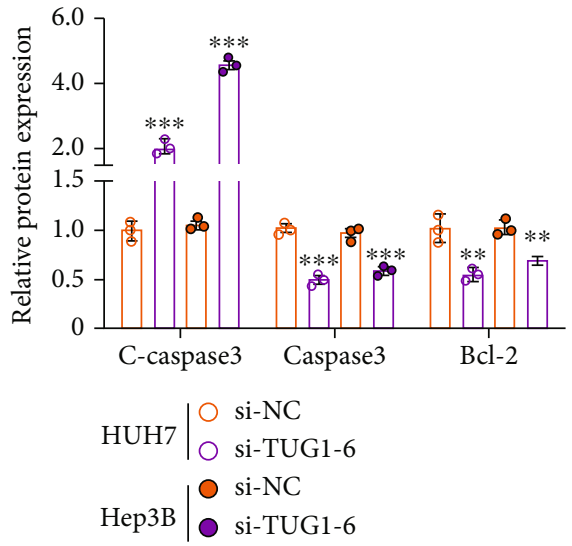

(v)

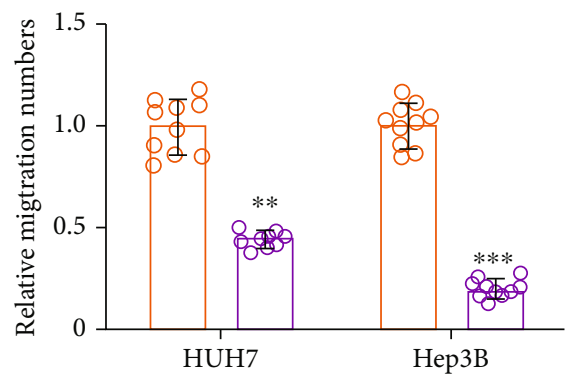

si-NC

si-TUG1-6

(x)

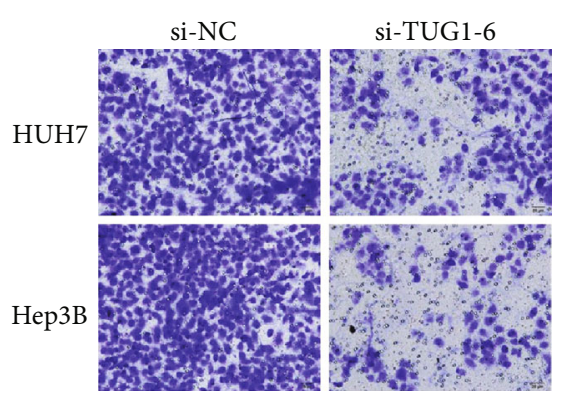

(w)

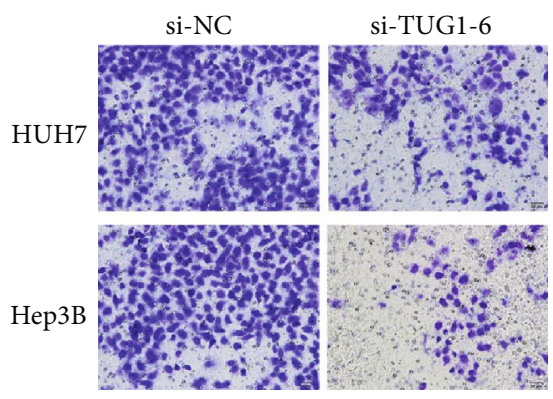

(y)

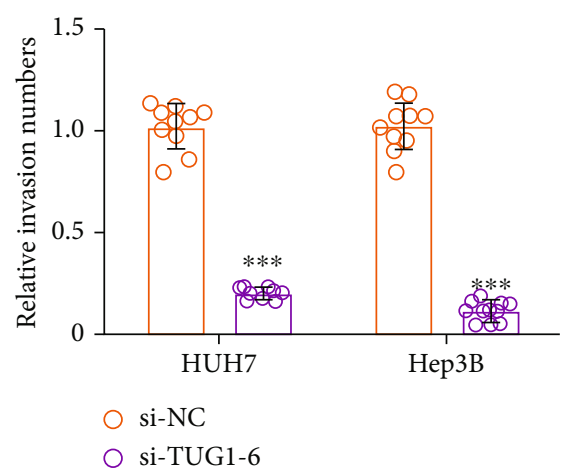

(z)

FIGURE 5: The antitumor activity of si-TUG1-6 in vitro. $(\mathrm{a}, \mathrm{b})$ The immunofluorescence results of HUH7-TUG1-IRES-EGFP and Hep3BTUG1-IRES-EGFP cells treated with si-TUG1-6. (c) TUG1 expression, (d) Siglec-15 mRNA, and (e, f) protein expression in HUH7 and Hep3B cells treated si-TUG1-6. (g, h) Relative luciferase activity of Jurkat-RGA in HUH7 and Hep3B cells treated with si-TUG1-6. (i, j) $\mathrm{T}$ cell-induced cytotoxicity cocultured with HUH7 and Hep3B cells treated with si-TUG1-6. (k, l) IFN- $\gamma$ and (m, n) IL-2 secreted by T cells from $(i, j)$. (o, p) Cell proliferation in HUH7 and Hep3B cells treated with si-TUG1-6 analyzed by CCK8. (q, r) Cell proliferation in HUH7 and Hep3B cells treated with si-TUG1-6 analyzed by EdU assay. (s, t) Cell apoptosis in HUH7 and Hep3B cells treated with siTUG1-6 analyzed by Annexin V/PI. (u,v) Apoptosis-relevant proteins level in HUH7 and Hep3B cells treated si-TUG1-6. (w-z) (q, r) Migration and $(\mathrm{s}, \mathrm{t})$ invasion of HUH7 and Hep3B cells treated with si-TUG1-6. The data are presented as the means \pm SD, $n=3$ replicates in $(\mathrm{a}-\mathrm{v}), n=10$ samples in $(\mathrm{w}-\mathrm{z}) .{ }^{*} \mathrm{P}<0.05,{ }^{* *} \mathrm{P}<0.01,{ }^{* * *} \mathrm{P}<0.005$. S15: Siglec-15.

WTP mice at the endpoint. We then detected CD3+ T cell activity isolated from PBMCs through a T cell cytotoxicity assay. Compared to fresh CD3 + T cells, there was a reduction in $\mathrm{CD} 3+\mathrm{T}$ cells and a higher cytotoxicity activity in CD3+ T cells from the WTP group (Figures $6(\mathrm{~g})$ and 6(h)). Similar results were also found for cytokine release, and there was no difference between fresh CD3+ $\mathrm{T}$ cells and CD3+ T cells from the KTP group (Figures 6(i)-6(l)). These results suggested that $\mathrm{CD} 3+\mathrm{T}$ cells from the KTP group may be exhausted due to Siglec-15 and other immune checkpoint molecules. In contrast, the good performance of $\mathrm{CD} 3+\mathrm{T}$ cells from the WTP group in the immune response in vitro may be attributed to removing Siglec-15-induced restrictions on $\mathrm{T}$ cell activity. 

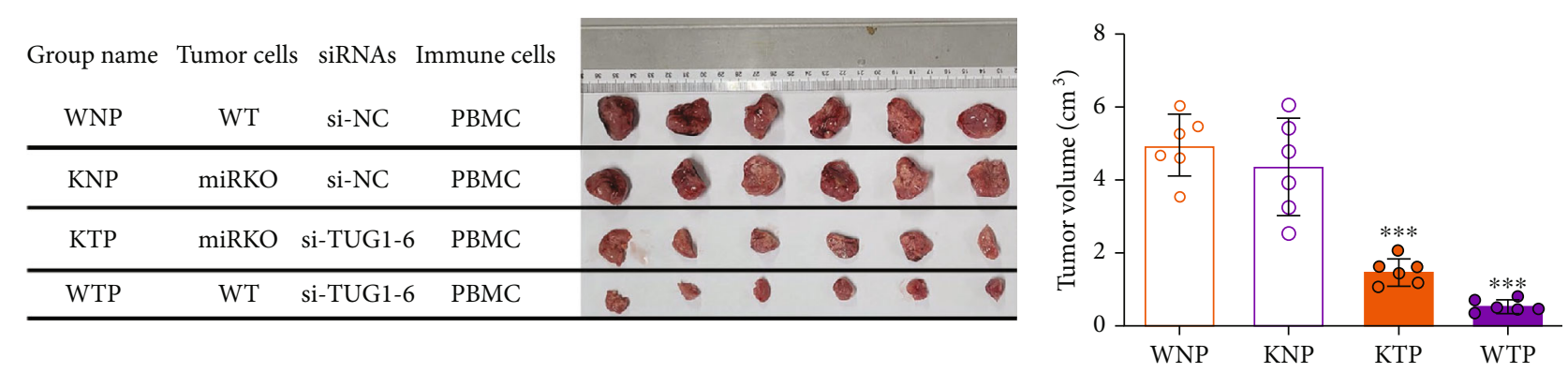

(a)

(b)
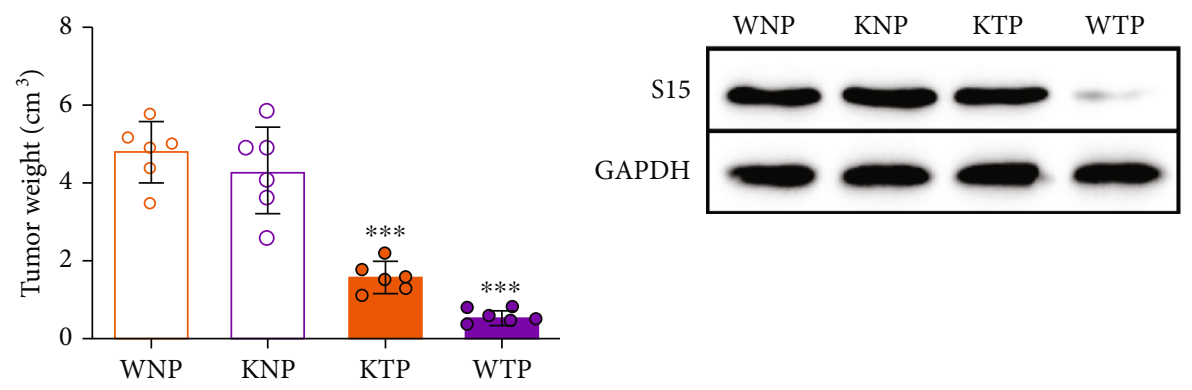

(c)

(d)
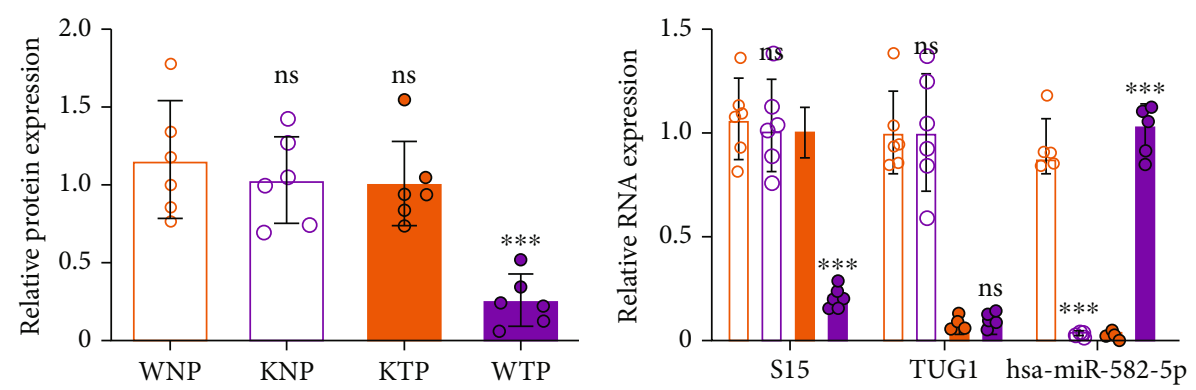
$\bigcirc$ WNP
O KTP
O KNP
○ WTP

(e)

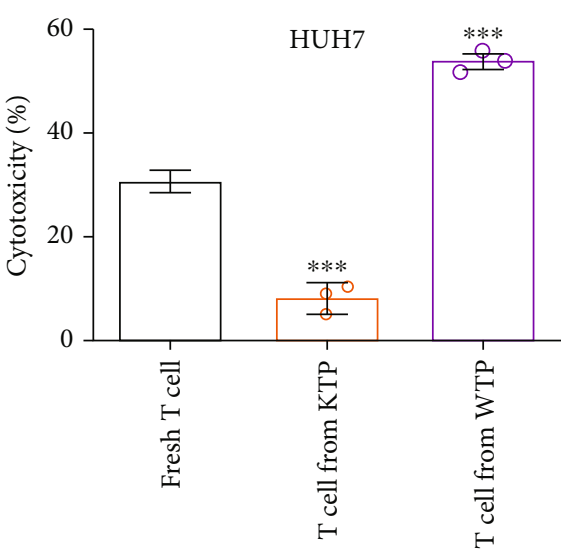

(g)

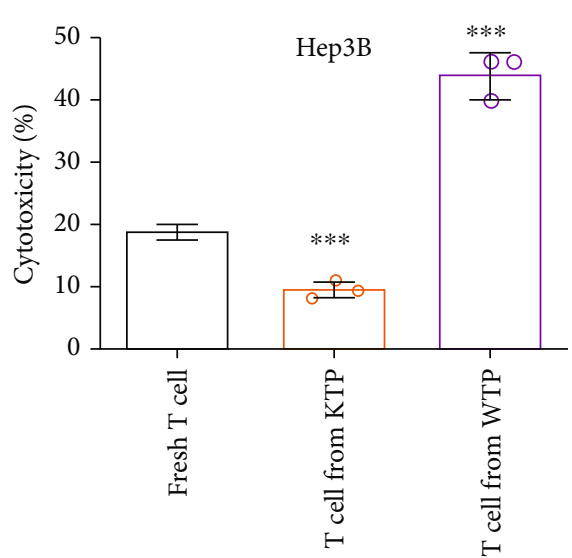

(h)

Figure 6: Continued. 


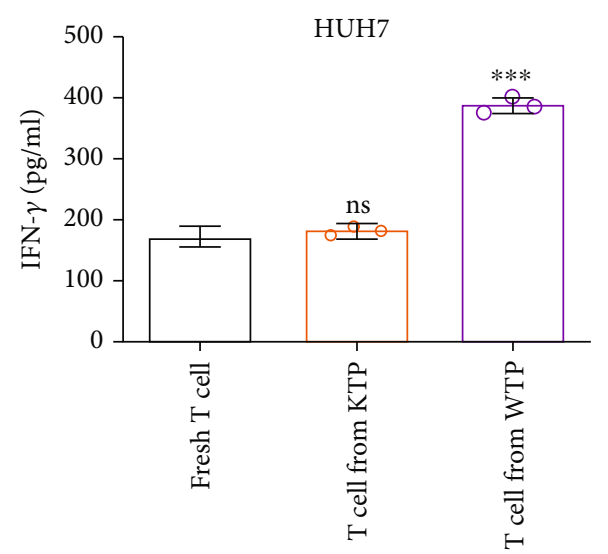

(i)

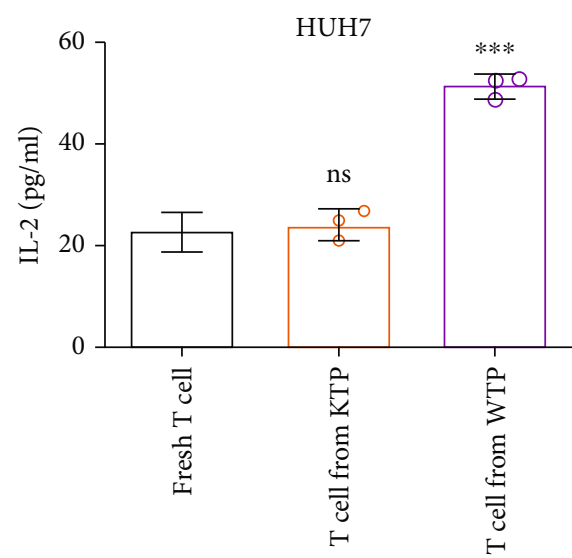

$(\mathrm{k})$

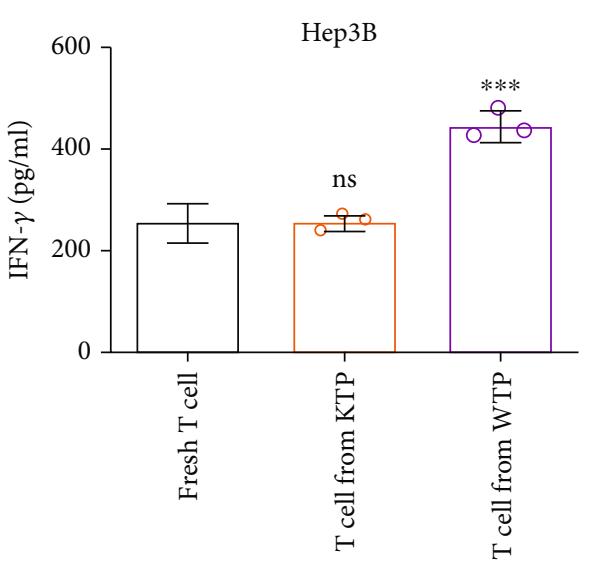

(j)

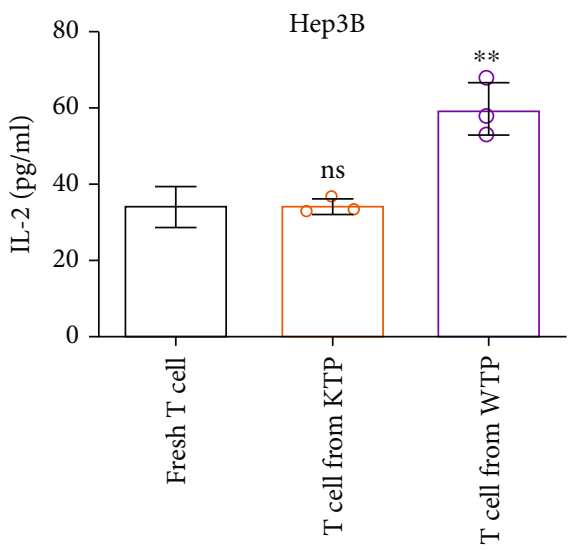

(1)

FIgURE 6: The antitumor activity of si-TUG1-6 in vivo. (a) The image of tumor growth the introduction of each group. WNP: NCG mice injected with wide-type Hep3B cells, si-NC, and human PBMC; KNP; NCG mice injected with Hep3B-miRKO cells, si-NC, and human PBMC; KTP: NCG mice injected with Hep3B-miRKO cells, si-TUG1-6, and human PBMC. WTP: NCG mice injected with wide-type Hep3B cells, si-TUG1-6, and human PBMC. (b) Tumor volume and (c) tumor wright in each group. (d, e) Siglec-15 protein expression in tumors from each group. (f) RNA expression of Siglec-15, hsa-miR-582-5p, and TUG1 in tumors from each group. (g, h) Cytotoxicity of T cells from mice in each groups and cocultured with HUH7 and Hep3B cells. (i, j) IFN- $\gamma$ and (k, l) IL-2 secreted by T cells from (g, h). The data are presented as the means $\pm \mathrm{SD}, n=6$ samples and 3 replicates in $(\mathrm{b}-\mathrm{f}), n=3$ replicates in $(\mathrm{g}-\mathrm{k}) .{ }^{*} P<0.05,{ }^{* *} P<0.01,{ }^{* * *}$ $P<0.005$. S15: Siglec-15.

Together, these in vivo results demonstrated that siTUG1-6 displays antitumor activity in HCC with a synergetic effect by inhibiting tumor progression and increasing immune activity via repressing Siglec-15 expression.

\section{Discussion}

Antibodies have become one of the most effective pharmaceuticals for treating human diseases, including cancers. Among the antibody drugs for treating cancers, one type of drug targeting immune checkpoints displays promising therapeutic effects on many cancers, such as lung cancer or breast cancer [17]. Many immune checkpoint antigens are used in antibody drugs, such as PD1/PD-L1, LAG-3, and CTLA-4/CD80/CD86 [17]. These antibody drugs increase the immune response to cancers by blocking cancerinduced immunosuppression in the body after binding to immune checkpoint antigens. Thus, a substitute strategy may work to achieve the same purpose by knocking down immune checkpoint expression using siRNAs [18]. Lian et al. treated lung metastasis using an epithelial cell adhesion molecule- (EpCAM-) targeted cationic liposome (LPP-P4Ep) containing si-CD47 and si-PD-L1; they found that lung metastasis was reduced in the lung metastasis model [19].

Siglec-15 was initially characterized by Dr. Takashi Angata in 2007 as a unique member that is selectively expressed on myeloid cells and osteoclasts (a bone-specific myeloid lineage) but generally absent in other immune cells and tissues $[5,7,20]$. In 2019, the Lieping Chen group stated that Siglec-15 is an immune checkpoint comparable to PDL1 with high expression in macrophages and many cancers, including liver cancers $[7,21]$. Thus, Siglec-15 is called a next-generation immunooncology agent and has received much attention, especially in antibody drug development. However, the regulatory mechanism of Siglec-15 in cancers remains unclear.

Many studies have shown that TUG1 plays a significant role in tumor progression. Zhang et al. found that 
downregulation of TUG1 inhibits cell proliferation and promotes apoptosis in osteosarcoma [11]. Shao et al. showed that knockdown of TUG1 suppresses cell proliferation and migration through the KLF4/miR-153-1 axis [13]. Notably, TUG1 has also been demonstrated to promote cell migration, invasion, and proliferation in HCC through different pathways, such as the miR-29c-3p/COL1A1 axis [10], miR137/AKT2 axis [22], and miR-144/JAK2/STAT3 axis [16]. However, the understanding of TUG1 in immune response regulation remains unknown. We first demonstrated the regulatory role of TUG1 in the immune response by upregulating the Siglec-15 level in HCC cells, aiding in the establishment of immunosuppression. These findings suggested that TUG1 is a dual-function target for RNAi drug development.

Cancer treatments composed of immune checkpoint inhibitors and oncogene-targeted drugs display a better therapeutic effect in clinical conditions, such as lung cancer, breast cancer, and colon cancer [23]. This strategy includes the following two antitumor mechanisms: inhibiting tumor progression and blocking immunosuppression. However, two or more drugs are needed to achieve this purpose, resulting in a greater possibility of toxicity and side effects. Thus, a single molecule with double antitumor functions may be safer and more effective. Our study demonstrated that TUG1 is an appropriate target because its knockdown displayed dual antitumor functions. We also developed an efficient siRNA targeting TUG1 (si-TUG1-6), which exhibited antitumor activity in vitro and in vivo. Combined with the GalNAc-siRNA conjugate technique, which has high safety and high delivery efficiency into hepatocytes in vivo [24-26], our study suggests that TUG1 is an appropriate target to treat HCC through siRNA drugs.

In conclusion, TUG1 plays a regulatory role in Siglec-15 expression in HCC cells by targeting and sponging hsa-miR582-5p. TUG1-induced Siglec-15 upregulation increases the immunosuppression between HCC cells and T cells. Moreover, siRNA downregulation of TUG1 expression shows antitumor activity in HCC cells by suppressing tumor progression, such as proliferation, migration, and invasion, as well as by enhancing the $\mathrm{T}$ cell-induced immune response both in vitro and in vivo. These findings indicated that TUG1 is a potential and applicable target for RNAi drug development in HCC treatment.

\section{Data Availability}

The data used to support the findings of this study are available from the corresponding authors upon reasonable request.

\section{Conflicts of Interest}

None of the authors has any competing interests in the manuscript.

\section{Authors' Contributions}

LH and YY conceived and designed the experiments. YR, JL, and YG analyzed the data. YG, YY, and LH wrote the paper.
All authors read and approved the final manuscript, Yanyi Ren, Jiayi Lyu, and Yaoguang Guo contributed equally to this work and are co-first authors.

\section{References}

[1] J. Ferlay, I. Soerjomataram, R. Dikshit et al., "Cancer incidence and mortality worldwide: sources, methods and major patterns in GLOBOCAN 2012," International Journal of Cancer, vol. 136, no. 5, pp. E359-E386, 2015.

[2] E. G. Giannini, F. Farinati, F. Ciccarese et al., "Prognosis of untreated hepatocellular carcinoma," Hepatology, vol. 61, no. 1, pp. 184-190, 2015.

[3] R. L. Setten, J. J. Rossi, and S. P. Han, "The current state and future directions of RNAi-based therapeutics," Nature Reviews Drug Discovery, vol. 18, no. 6, pp. 421-446, 2019.

[4] A. D. Springer and S. F. Dowdy, "GalNAc-siRNA conjugates: leading the way for delivery of RNAi therapeutics," Nucleic Acid Therapeutics, vol. 28, no. 3, pp. 109-118, 2018.

[5] T. Angata, Y. Tabuchi, K. Nakamura, and M. Nakamura, "Siglec-15: an immune system Siglec conserved throughout vertebrate evolution," Glycobiology, vol. 17, no. 8, pp. 838846, 2007.

[6] C. Pan, H. Liu, E. Robins et al., "Next-generation immunooncology agents: current momentum shifts in cancer immunotherapy," Journal of Hematology \& Oncology, vol. 13, no. 1, p. $29,2020$.

[7] J. Wang, J. Sun, L. N. Liu et al., "Siglec-15 as an immune suppressor and potential target for normalization cancer immunotherapy," Nature Medicine, vol. 25, no. 4, pp. 656-666, 2019.

[8] J. J. Quinn and H. Y. Chang, "Unique features of long noncoding RNA biogenesis and function," Nature Reviews Genetics, vol. 17, no. 1, pp. 47-62, 2016.

[9] T. L. Young, T. Matsuda, and C. L. Cepko, "The noncoding RNA taurine upregulated gene 1 is required for differentiation of the murine retina," Current Biology, vol. 15, no. 6, pp. 501$512,2005$.

[10] W. Zhao, X. Jiang, and S. Yang, "IncRNA TUG1 promotes cell proliferation, migration, and invasion in hepatocellular carcinoma via regulating miR-29c-3p/COL1A1 axis," Cancer Management and Research, vol. Volume 12, pp. 6837-6847, 2020.

[11] Q. Zhang, P. L. Geng, P. Yin, X. L. Wang, J. P. Jia, and J. Yao, "Down-regulation of long non-coding RNA TUG1 inhibits osteosarcoma cell proliferation and promotes apoptosis," Asian Pacific Journal of Cancer Prevention, vol. 14, no. 4, pp. 2311-2315, 2013.

[12] Y. Han, Y. Liu, Y. Gui, and Z. Cai, "Long intergenic noncoding RNA TUG1 is overexpressed in urothelial carcinoma of the bladder," Journal of Surgical Oncology, vol. 107, no. 5, pp. 555-559, 2013.

[13] H. Shao, D. Dong, and F. Shao, "Long non-coding RNA TUG1-mediated down-regulation of KLF4 contributes to metastasis and the epithelial-to-mesenchymal transition of colorectal cancer by miR-153-1," Cancer Management and Research, vol. 11, pp. 8699-8710, 2019.

[14] Z. Cheng, C. Yu, S. Cui et al., "c ircTP63 functions as a ceRNA to promote lung squamous cell carcinoma progression by upregulating FOXM1," Nature Communications, vol. 10, no. 1, p. 3200, 2019.

[15] T. C. Shih, Y. J. Tien, C. J. Wen et al., "MicroRNA-214 downregulation contributes to tumor angiogenesis by inducing 
secretion of the hepatoma-derived growth factor in human hepatoma," Journal of Hepatology, vol. 57, no. 3, pp. 584591, 2012.

[16] J. Lv, Y. Kong, Z. Gao, Y. Liu, P. Zhu, and Z. Yu, "LncRNA TUG1 interacting with miR-144 contributes to proliferation, migration and tumorigenesis through activating the JAK2/ STAT3 pathway in hepatocellular carcinoma," The International Journal of Biochemistry \& Cell Biology, vol. 101, pp. 19-28, 2018.

[17] W. Chen, Y. Yuan, and X. Jiang, "Antibody and antibody fragments for cancer immunotherapy," Society, vol. 328, pp. 395406, 2020.

[18] F. Mainini and M. R. Eccles, "Lipid and polymer-based nanoparticle siRNA delivery systems for cancer therapy," Molecules, vol. 25, no. 11, p. 2692, 2020.

[19] S. Lian, R. Xie, Y. Ye et al., "Simultaneous blocking of CD47 and PD-L1 increases innate and adaptive cancer immune responses and cytokine release," eBioMedicine, vol. 42, pp. 281-295, 2019.

[20] M. Stuible, A. Moraitis, A. Fortin et al., "Mechanism and function of monoclonal antibodies targeting Siglec-15 for therapeutic inhibition of osteoclastic bone resorption," The Journal of Biological Chemistry, vol. 289, no. 10, pp. 64986512, 2014.

[21] J. Lou, Y. Hao, K. Lin et al., "Circular RNA CDR1as disrupts the p53/MDM2 complex to inhibit gliomagenesis," Molecular Cancer, vol. 19, no. 1, p. 138, 2020.

[22] W. Li, J. Ge, J. Xie, J. Yang, J. Chen, and T. He, "LncRNATUG1 promotes hepatocellular carcinoma migration and invasion via targeting the miR-137/AKT2 axis," Cancer Biotherapy and Radiopharmaceuticals, vol. 36, no. 10, pp. 850-862, 2021.

[23] L. M. Colli, M. J. Machiela, H. Zhang et al., "Landscape of combination immunotherapy and targeted therapy to improve cancer management," Cancer Research, vol. 77, no. 13, pp. 3666-3671, 2017.

[24] S. Matsuda, K. Keiser, J. K. Nair et al., "siRNA conjugates carrying sequentially assembled trivalent $\mathrm{N}$-acetylgalactosamine linked through nucleosides elicit robust gene silencing in vivo in hepatocytes," ACS Chemical Biology, vol. 10, no. 5, pp. 1181-1187, 2015.

[25] M. M. Janas, I. Zlatev, J. Liu et al., "Safety evaluation of $2^{\prime}$ -deoxy-2' -fluoro nucleotides in GalNAc-siRNA conjugates," Nucleic Acids Research, vol. 47, no. 7, pp. 3306-3320, 2019.

[26] D. J. Foster, C. R. Brown, S. Shaikh et al., "Advanced siRNA designs further improve in vivo performance of GalNAcsiRNA conjugates," Molecular Therapy, vol. 26, no. 3, pp. 708-717, 2018. 